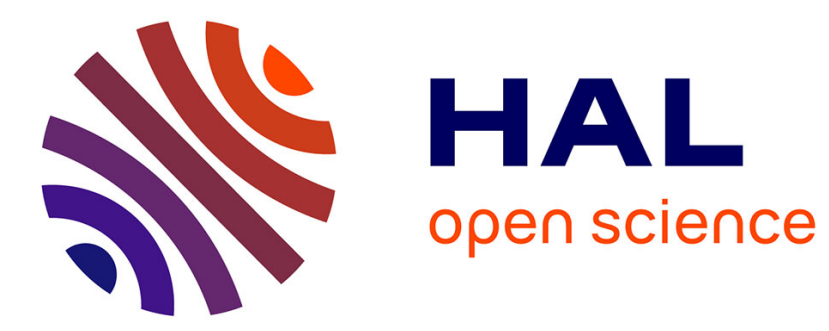

\title{
Paradoxical evolution of rickettsial genomes
}

\author{
Awa Diop, Didier Raoult, Pierre-Edouard Fournier
}

\section{To cite this version:}

Awa Diop, Didier Raoult, Pierre-Edouard Fournier. Paradoxical evolution of rickettsial genomes. Ticks and Tick-borne Diseases, 2019, 10 (2), pp.462-469. 10.1016/j.ttbdis.2018.11.007 . hal-02101496

\section{HAL Id: hal-02101496 \\ https://hal-amu.archives-ouvertes.fr/hal-02101496}

Submitted on 21 Oct 2021

HAL is a multi-disciplinary open access archive for the deposit and dissemination of scientific research documents, whether they are published or not. The documents may come from teaching and research institutions in France or abroad, or from public or private research centers.
L'archive ouverte pluridisciplinaire HAL, est destinée au dépôt et à la diffusion de documents scientifiques de niveau recherche, publiés ou non, émanant des établissements d'enseignement et de recherche français ou étrangers, des laboratoires publics ou privés.

\section{다)(1) $\$$}

Distributed under a Creative Commons Attribution - NonCommercial| 4.0 International 


\section{Paradoxical evolution of rickettsial genomes}

Awa Diop ${ }^{1}$, Didier Raoult ${ }^{2}$ and Pierre-Edouard Fournier ${ }^{1 *}$

${ }^{1}$ UMR VITROME, Aix-Marseille University, IRD, Service de Santé des Armées, Assistance Publique-Hôpitaux de Marseille, Institut Hospitalo-Uuniversitaire Méditerranée Infection, 1921 Boulevard Jean Moulin, 13005 Marseille, France Tel: +33 413732 401, Fax: +33 413732 402.

${ }^{2}$ UMR MEPHI, Aix-Marseille University, IRD, Assistance Publique-Hôpitaux de Marseille, Institut Hospitalo-Uuniversitaire Méditerranée Infection, Marseille, France

*Corresponding author: Pr Pierre-Edouard Fournier

Email: pierre-edouard.fournier@univ-amu.fr 


\section{Abstract:}

1 Rickettsia species are strictly intracellular bacteria that evolved approximately 150 million

2 years ago from a presumably free-living common ancestor from the order Rickettsiales that

3 followed a transition to an obligate intracellular lifestyle. Rickettsiae are best known as

4 human pathogens vectored by various arthropods causing a range of mild to severe human

5 diseases. As part of their obligate intracellular lifestyle, rickettsial genomes have undergone a

6 convergent evolution that includes a strong genomic reduction resulting from progressive

7 gene degradation, genomic rearrangements as well as a paradoxical expansion of various

8 genetic elements, notably small RNAs and short palindromic elements whose role remains

9 unknown. This reductive evolutionary process is not unique to members of the Rickettsia genus but is common to several human pathogenic bacteria. Gene loss, gene duplication,

DNA repeat duplication and horizontal gene transfer all have shaped rickettsial genome evolution. Gene loss mostly involved amino-acid, ATP, LPS and cell wall component biosynthesis and transcriptional regulators, but with a high preservation of toxin-antitoxin (TA) modules, recombination and DNA repair proteins. Surprisingly the most virulent Rickettsia species were shown to have the most drastically reduced and degraded genomes compared to closely related species of milder pathogenesis. In contrast, the less pathogenic species harbored the greatest number of mobile genetic elements. Thus, this distinct evolutionary process observed in Rickettsia species may be correlated with the differences in virulence and pathogenicity observed in these obligate intracellular bacteria. However, future investigations are needed to provide novel insights into the evolution of genome sizes and content, for that a better understanding of the balance between proliferation and elimination of genetic material in these intracellular bacteria is required.

Keywords: Rickettsia, genomics, evolution, virulence, genome rearrangement, non-coding DNA, gene loss, DNA repeats 


\section{Introduction}

The genus Rickettsia (order Rickettsiales, family Rickettsiaceae) comprises strictly intracellular $\alpha$-proteobacteria mostly associated with diverse arthropod vectors around the world (Raoult and Roux, 1997; Stothard et al., 1994). Rickettsia species evolved approximately 150 million years ago from a common ancestor of Rickettsiales that was presumably free-living, and progressively followed a transition to an obligate intracellular lifestyle that occurred 775-525 million years ago and then to primarily infecting arthropod lineages approximately 525-425 million years ago (El Karkouri et al., 2016; Merhej and Raoult, 2011; Lucy A Weinert et al., 2009). These bacteria are also well known to infect mammalian hosts, mostly through arthropod bites or arthropod feces infecting scratching lesions. On the basis of their phenotypic properties, vector hosts and phylogenetic organization, Rickettsia species were split into three to four groups by different authors (Figure 1): i) the spotted fever group (SFG, Figure 1) contains many spotted fever-causing species as well as numerous species of as-yet unknown pathogenicity. SFG rickettsiae are mostly associated with ticks, but also fleas and mites (Diop et al., 2017); ii) the second phylogenetic group, the typhus group (TG, Figure 1) is only made of $R$. prowazekii and $R$. typhi that cause epidemic and murine typhus, and are associated with human body lice and rat fleas, respectively (Diop et al., 2017); iii) the ancestral group includes $R$. bellii and $R$. canadensis. These species diverged early from SFG and TG rickettsiae, are associated with ticks but do not cause human disease (Figure 1) (Diop et al., 2017); iv) a fourth group, named transitional group, was proposed by Gillespie et al. to include SFG species phylogenetically close to R. felis (Gillespie et al., 2007). However, as these species do not exhibit significant differences with other SFG species except their phylogenetic position, several authors discussed the validity of this latter group (Shpynov et al., 2018). 
Rickettsia species cause a range of illnesses, from mild and self-limiting to severe and life-threatening diseases (Diop et al., 2017). Currently, the most common rickettsioses are African tick-bite fever caused by $R$. africae, scalp eschar and neck lymphadenopathy (SENLAT) caused by $R$. slovaca, Mediterranean spotted fever (MSF) caused by $R$. conorii, Rocky Mountain spotted fever (RMSF) caused by $R$. rickettsii and murine typhus caused by R. typhi. (El Karkouri et al., 2017; Parola et al., 2013; Sahni et al., 2013). Rickettsia prowazekii, the historical agent of epidemic typhus, is only rarely encountered currently but has a strong epidemic potential (Parola et al., 2013). Furthermore, recent studies have reported the association of other Rickettsia lineages with other reservoirs including protozoa, algae, leeches, plants or insects (Merhej and Raoult, 2011; Murray et al., 2016; Weinert et al., 2009).

In 1998, the first complete Rickettsia genome, that of $R$. prowazekii strain Madrid E, was sequenced (Andersson et al., 1998). It was the seventh bacterial genome to be sequenced. Subsequently, the genomes of many Rickettsia species have been fully sequenced, allowing a better knowledge of the molecular mechanisms involved in their pathogenicity (Balraj et al., 2009). Genome sequencing also appeared as a potential tool to revolutionize the phylogenetic and evolutionary investigations of prokaryotes, especially endosymbiotic bacteria. Hence, deciphering rickettsial genomes appeared as an efficient tool to understand the evolution of these obligate intracellular bacteria.

\section{General features of rickettsia genomes}

Rickettsia species have small genome sizes and low $\mathrm{G}+\mathrm{C}$ contents. SFG and TG rickettsiae exhibit genome sizes of 1.25 to $2.3 \mathrm{Mb}$, and $1.11 \mathrm{Mb}$, respectively. They also exhibit $\mathrm{G}+\mathrm{C}$ contents ranging from 32.2 to $33.0 \%$ and 28.9 to $29.0 \%$, respectively. Rickettsia species have numbers of predicted protein-coding genes varying between 817 and 2,479 and most of them 
maintain a near perfect chromosomal synteny (Diop et al., 2017), which enabled the identification of an ongoing and progressive genome degradation (Ogata, 2001). Rickettsial genomes contain many functional or unfunctional pseudogenes and possess a high percentage of non-coding DNA (Blanc et al., 2007; McLeod et al., 2004) (Fig. 2). This percentage of non-coding DNA ranges from $16.2 \%$ for $R$. felis to $31 \%$ for $R$. massiliae. Rickettsia prowazekii, the most reduced rickettsial genome contains $24 \%$ of non-coding sequence. By comparison, Chlamydia trachomatis, another strictly intracellular bacterium, possesses only 10\% non-coding DNA (Andersson et al., 1998; Holste et al., 2000; Rogozin et al., 2002).This pseudogenization progressively leads to a genome downsizing and results from a switch from a free-living to an obligate intracellular lifestyle. This progressive reductive evolution has allowed rickettsiae to purge unnecessary and redundant genes mainly involved in metabolisms supplied by eukaryotic host cells (Georgiades and Raoult, 2011; Merhej et al., 2009). Paradoxically to this ongoing genomic reduction, rickettsial genomes exhibit another marker of convergent evolution, $i$. e., the expansion of genetic elements including small RNAs, tandem repeats, short palindromic elements named rickettsia palindromic elements (RPEs) (Ogata et al., 2002), ankyrin and tetratricopeptide repeats and gene family duplication mainly ADP-ATP translocases, toxin-antitoxin modules and type IV secretion system (T4SS). Another unexpected property of rickettsial genomes is the presence of plasmids, the first described in obligate intracellular bacteria. The first plasmid was identified in R. felis (Ogata et al., 2005a). To date, at least 20 rickettsial plasmids have been described in 11 species. Their number varies from 1 to 4 per species/strain (Baldridge et al., 2007; G. Blanc et al., 2007; El Karkouri et al., 2016). These findings suggest possible exchanges of genetic material by conjugation, a mechanism that was thought to be absent in obligate intracellular and allopatric bacteria (Georgiades and Raoult, 2011; Merhej et al., 2009; Ogata et al., 2005a). 


\section{Rickettsia genome in an ongoing convergent evolution}

\subsection{Ongoing reductive evolution of Rickettsial genomes}

Following their adaptation from a free-living to an obligate intracellular lifestyle in eukaryotic cells, rickettsiae underwent genomic changes to fit their specific bottleneck ecosystem, resulting not only in a reducing genome size but also in a specific genomic architecture (Keeling et al., 1994; Sicheritz-Pontén and Andersson, 1997). Comparative genomics revealed that rickettsiae, by taking advantage of host cell metabolites, underwent a genome reductive evolution (Georgiades and Raoult, 2011; Merhej et al., 2009) that occurred through a progressive pseudogenization (Fig. 2) and gene loss of selected biosynthetic pathway components (Andersson et al., 1998; Audia and Winkler, 2006; Fournier et al., 2009; Ogata, 2001; Sakharkar, 2004; Walker, 2005; Wolf and Koonin, 2013). In addition, genomic degradation was detrimental for the $\mathrm{G}+\mathrm{C}$ content, as it led to an enrichment in $\mathrm{A}+\mathrm{T}$, in particular in the high proportion of non-coding DNA (Sakharkar, 2004). However, a great variation in chromosome size, ranging from 1.1 to $2.3 \mathrm{Mb}$, is observed in rickettsiae (Diop et al., 2017), indicating that some species are at a more advanced stage of reductive genomic evolution (TG rickettsiae) than others (SFG rickettsiae) (Ogata, 2001). In ehrlichiae, a similar genomic reduction is observed, but the $\mathrm{G}+\mathrm{C}$ content may remain as high as $49.8 \%$ in Anaplasma species (Dunning Hotopp et al., 2006), suggesting that the reductive process in these bacteria had a lesser impact on the $\mathrm{G}+\mathrm{C}$ content degradation. Rickettsial genomes are characterized by a high rate of accumulation of slightly harmful deletions, mutations and insertions (Brynnel et al., 1998). Alternatively, gene loss can also result from the accumulations of small mutations. The formation of internal stop codons within intact genes can occur through the creation of a frameshit by single base mutation, insertion or deletion (Ogata, 2001). This induces the genome degradation resulting from fragmented gene accumulation or gene remnants. An unexpected finding of rickettsial genomics was that the 
most virulent species had the most reduced genomes (Fournier et al., 2009). Such a finding is not an isolated phenomenon as in Mycobacterium, Streptococcus spp., Corynebacterium spp. and other genera, the highest degree of gene loss is observed in the most virulent species when compared to closely related and milder or nonpathogenic species (Blanc et al., 2007; Merhej et al., 2013; Ogata, 2001). Many of the genes required by free-living bacteria are absent in Rickettsia (Bechah et al., 2010) and degraded genes include mostly those involved in the biosynthesis of nutrients (Blanc, 2005; Ogata, 2001; Renesto et al., 2005). For example, Rickettsia exhibits few genes for de novo nucleotide synthesis, $i$. e., only those for conversion of nucleoside monophosphates into all other nucleotides, implying that they take up nucleoside monophosphates from the host (Wixon, 2001). Analysis of $R$. conorii and $R$. prowazekii genomes (Dunning Hotopp et al., 2006; Ogata, 2001) revealed that genes coding glycolytic enzymes and those required for nucleotide or cofactor biosynthesis are totally absent in $R$. conorii and $R$. prowazekii when compared to most genera in the order Rickettsiales that have complete glycolytic pathways. Nevertheless, rickettsiae must obtain glycerol-3-phosphate from the host via a glycerol-3-phosphate transporter (Dunning Hotopp et al., 2006). This ATP production profile is similar for Rickettsia and mitochondria, as they possess a high number of ATP/ADP translocases, suggesting that they have both evolved from a common ancestor (Andersson et al., 1998; Renesto et al., 2005). In addition, the genome sequencing of $R$. prowazekii revealed a lack of amino acid metabolism such as those for glutamate metabolism (Andersson et al., 1998; Fuxelius et al., 2007). The enzymes involved in the aspartate and alanine metabolism pathways, and those playing a role in the biosynthesis of leucine, valine, isoleucine and aromatic amino acids (tryptophan, tyrosine, phenylalanine) are similarly missing in Rickettsia species (Renesto et al., 2005), suggesting the use of host-derived amino acids for their growth, survival and replication. Additionally, all Rickettsia species except $R$. belli have a reduced set of folate biosynthesis genes (Fuxelius et 
al., 2007). In TG rickettsiae all five genes required for the de novo folate biosynthesis are lacking (Hunter et al., 2015). Furthermore, a limited set of genes for LPS and cell wall component biosynthesis, including lipid-A and peptidoglycan, respectively, were identified in Rickettsia species (Fuxelius et al., 2007). The rickettsial surface protein-coding genes rickA and sca 2 are another example of genes that were degraded or eliminated by Rickettsia species during their specialization. The RickA protein participates in actin polymerization through the activation of Arp2/3 similar to that found in Listeria monocytogenes and Shigella spp. (Balraj et al., 2008b; Gouin et al., 2004, 1999). While lacking in the TG, rickA is present in all AG and SFG rickettsial genomes avalaible (Baldridge et al., 2005; Balraj et al., 2008a, 2008b; Heinzen et al., 1993; Jeng et al., 2004; McLeod et al., 2004; Ogata, 2001; Ogata et al., 2006, 2005a). The absence of rickA in $R$. prowazekii is not surprising if we consider its lack of actin motility. In contrast, $R$. typhi exhibits a unique and erratic actin-based motility despite having a nonfunctional RickA protein (McLeod et al., 2004; Reed et al., 2014). In addition, $R$. canadensis expresses RickA but does not exhibit actin-based motility (Heinzen et al., 1993). These data suggest the possible involvement of other actin polymerization mechanisms and that RickA alone may not be sufficient or required for actin-based rickettsial motility. Nevertheless, it was proposed that RickA originated early in rickettsial evolution and may have been lost during the divergence of the TG. Recent research suggests that Rickettsia spp. use also Sca2 for actin-based motility with a distinct mechanism compared to RickA. Sca2 was found to be intact in $R$. conorii, absent in $R$. prowazekii and pseudogenized in $R$. typhi (McLeod et al., 2004). In R. typhi, Sca2 lacks the FH1 (formin homology 1) domain and contains only a proline-rich tract and a series of five WH2 domains ( $\beta$-domains) in different locations with a divergence in sequences (Sears et al., 2012). The evolutionary process of genome degradation in rickettsiae led to loss of transcriptional regulator genes with a decreased translational capacity as observed in R. prowazekii (Andersson and Kurland, 1998), 
despite conserved gene sets coding for toxins, toxin-antitoxin (TA) modules and recombination and DNA repair proteins most likely needed for protection against host immune response (Moran, 2002).

The reductive evolution of rickettsial genomes is not only the consequence of gene degradation or loss, but it is also linked to a differential expression level of genes (Diop et al., 2017). Some genes under the influence of evolutionary forces are dormant or repressed while others under this effect are overexpressed. Recent research involving two virulent and two milder SFG rickettsiae demonstrated that the two virulent agents $R$. conorii (MSF) and $R$. slovaca (SENLAT) have the most reduced genome and displayed less up-regulated than down-regulated genes than the milder $R$. massiliae and $R$. raoultii causing MSF and SENLAT, respectively (El Karkouri et al., 2017), that have less reduced genomes.

Consequently, to adapt to their specific intracellular environment, Rickettsia species were shaped by distinct evolutionary processes. The most pathogenic species are characterized by a strong reductive genomic evolution, with a higher genome degradation rate and accumulation of non-coding DNA than less pathogenic species. These findings suggest that reductive genomic evolution, resulting in protein structural variations, is associated to the emergence of virulence (El Karkouri et al., 2017). It was speculated that the loss of regulator genes, as observed in several intracellular pathogens, is a critical cause of virulence (Darby et al., 2007). This reductive genomic evolution appears to have occurred in several other human pathogens that have no common intracellular ancestor with Rickettsia such as Treponema spp., Mycobacterium spp. or Yersinia spp (Merhej et al., 2009; Walker, 2005; Wixon, 2001). Overall, during the course of evolution, rickettsial genomes exhibit a trend toward gene loss rather than acquisition, but strong selective effects co-exist with functional duplication required for survival. 


\subsection{Gene order, recombination events and "junk DNA" in rickettsial genomes}

A comparison of 13 rickettsial genomes (Diop et al., 2017) demonstrated that they exhibit a highly conserved synteny and present few genomic rearrangements, except for $R$. bellii that exhibits little colinearity with other genomes, and $R$. felis that underwent several inversions. In addition, $R$. typhi, underwent a $35-\mathrm{kb}$ inversion close to the replication terminus and a specific 124-kb inversion nearby the origin of replication when compared to $R$. prowazekii and $R$. conorii (McLeod et al., 2004). As in other bacteria, inversions that occured in the origin of replication region are also found in $R$. australis, $R$. helvetica and $R$. honei (X. Dong et al., 2012; Xin Dong et al., 2012; Xin et al., 2012), indicating that this region constitutes a hotspot for genomic rearrangement (Eisen et al., 2000). Homologous intra-chromosomal recombination, the principal mechanism for genomic rearrangement in rickettsiae, occured between repeated sequences or by site-specific recombination. Consequently, duplications, deletions and inversions arose through these structures (Andersson and Kurland, 1998; Krawiec and Riley, 1990). Such events have been observed in Rickettsia spp., in the so-called super-ribosomal protein gene operon. Highly conserved in a broad range of bacteria and archaea, this operon consists of about 40 genes located in seven operons in the same order (Sicheritz-Pontén and Andersson, 1997). Despite their conserved order in many bacteria including E. coli and Bacillus subtilis, genes in the ribosomal protein gene operon are scattered around the genomes of Haemophilus influenzae, Mycoplasma genitalium and $R$. prowazeki (Andersson and Kurland, 1998; Fraser et al., 1995). Ribosomal RNA genes in bacterial genomes are normally organized into an operon with a conserved order $16 \mathrm{~S}-23 \mathrm{~S}-5 \mathrm{~S}$, and tRNA genes are often found in the spacer between the $16 \mathrm{~S}$ and the $23 \mathrm{~S}$ rRNA genes (Krawiec and Riley, 1990). However, an unusual arrangement of rRNA genes has been observed in all avalaible Rickettsia genomes, as the 16S rRNA gene is separated from the 23S and 5S rRNA gene cluster (Andersson et al., 1999; Munson et al., 1993). A similar 
organization is observed in all members of the order Rickettsiales (Dunning Hotopp et al., 2006). The upstream spacer of the rearranged 23S rRNA gene in some Rickettsia species contains short repetitive sequences that have been eliminated in other related species, suggesting that the rearrangement of rRNA genes occurred by intra-chromosomal recombination prior to speciation in Rickettsia spp. Rickettsial genome analysis highlighted a second major genomic rearrangement in rickettsiae, the elongation factor proteins (tuf and fus) being present in more than one copy in Rickettsia genomes (Syvänen et al., 1996). These genes can serve as repeat sequences, and initiate a rapid gene loss through intra-chromosomal recombination (Krawiec and Riley, 1990). In addition, the degree and positions of deletions caused by intra-chromosomal recombination in Rickettsia is different among the species, which suggests that the homologous recombination is an ongoing process that may result in an ongoing genes loss under weak or no selection pressure.

When compared to other bacterial genomes, rickettsial genomes have a high percentage of non-coding DNA sequences which also contains many DNA repeat sequences (Holste et al., 2000; Rogozin et al., 2002). Non-coding DNA in rickettsial genomes is traditionally considered as "junk DNA" resulting from gene degradation. $R$. prowazekii and $R$. typhi, the most reduced rickettsial genomes, harbor high rates of non-coding DNA with 24.6 and $23.7 \%$, respectively. However, $R$. bellii exhibits the lowest rickettsial level of non-coding DNA with 14.8\% (Diop et al., 2017).

\subsection{Paradoxical genomic expansions}

From a general point of view, rickettsial genomes are typical of those of symbiotic bacteria, in which the reductive trend is the dominant mode of evolution (Andersson and Andersson, 1999; Georgiades and Raoult, 2011; Merhej et al., 2009; Ogata, 2005). However, 
despite this reductive evolution, a paradoxical expansion of genetic elements can still occur in rickettsial genomes (Ogata et al., 2002). Genome sequence analysis revealed that rickettsial genome expansion may occur through proliferation of selfish DNA (small non coding RNAs (sRNAs) and rickettsia palindromic elements (RPEs)), gene duplications and horizontal gene transfer (Merhej and Raoult, 2011). Bacterial non-coding RNAs, whose biogenesis is predominantly attributed to either the intergenic regions (trans-acting) or to the antisense strand of an open reading frame (cis-acting) (Schroeder et al., 2015), were well documented in many bacterial taxa including Enterobacteriaceae, Listeria monocytogenes, Clostridium perfringens, Staphylococcus aureus, Pseudomonas aeruginosa and Mycobacterium tuberculosis (Papenfort and Vanderpool, 2015). sRNAs are classified among the most important post-transcriptional regulators involved in virulence and adaptation depending on the host niche, through transcriptomic regulation (Schroeder et al., 2015). Schroeder et al. (2015) were the first to identify sRNAs in Rickettsia species. Twenty to $30 \%$ of intergenic regions presumably encode for trans-acting sRNAs (14 to 191 sRNAs, depending on species). These findings may explain the highly conserved intergenic spacers identified by early comparative studies in Rickettsia (Ogata, 2001). More than 1,700 trans-acting sRNAs were predicted in 16 genomes of 13 species spanning all rickettsial groups (Schroeder et al., 2015). Rickettsia prowazekii was shown to possess stem loop structures after homopolymeric $\operatorname{poly}(\mathrm{T})$ stretches in the termination sites where the expression of sRNAs occurs (Woodard and Wood, 2011). Rickettsia palindromic elements (RPEs) were identified in 2002 by Ogata et al. (Ogata et al., 2002). These genetic elements are more abundant in SFG than TG rickettsiae (Figure 2). In the $R$. conorii genome, a total of 656 RPEs, classified into 8 families, were identified (RPE-1 to RPE-8) and represent 3.2\% of the entire genome (Ogata et al., 2002). By comparison, only 10 of the 44 RPE-1 copies described in $R$. conorii were found in the $R$. prowazekii genome. Surprisingly, nine of these $10 \mathrm{RPE}-1$ copies that are present in $R$. 
prowazekii are inserted in protein-coding genes, versus 19/44 in $R$. conorii. In addition, the RPE-1s inserted into protein-coding genes have a position compatible with the 3-dimentional fold and function of proteins (Ogata et al., 2000). This process of genomic evolution by inserting RPEs within protein-coding genes was initially thought to be unique to Rickettsia species but is also encountered in the Wolbachia genus (Ogata et al., 2005b; Riegler et al., 2012). Bacteria may use this random strategy to adapt their genetic repertoire in response to selective environmental pressure. The presence of a mobile element inserted in many unrelated genes also suggests the potential role of selfish DNA in rickettsial genome for de novo creation of new protein sequences during the course of evolution, suggesting an implication in the dynamics of genome evolution (Claverie and Ogata, 2003). Moreover, genomic comparison also enabled the identification of several copies of Ankyrin and Tetratricopeptide (TPR)-repeats in rickettsiae. Such repeated elements are frequently found in endosymbionts and assumed to play a role in host-pathogen interaction (Caturegli et al., 2000; Felsheim et al., 2009; Seshadri et al., 2003; Wu et al., 2004). Twenty-two copies of ankyrinand 11 copies of TPR-repeats were found in R. felis (Ogata et al., 2005a). In both species, they were proposed to be linked to pathogenicity. In Legionella pneumophila, which exhibits 20 Ankyrin-repeat copies and numerous TPR-repeat copies, these elements are suspected to play a modulatory role in the interactions with the host cytoskeleton and in interferences with the host cell trafficking events, respectively (Cazalet et al., 2004).

In addition to DNA repeat sequences, various gene families are duplicated in rickettsial genomes. Gene duplication was considered as an important source of bacterial adaptation to environmental changes in the host (Hooper, 2003). Following duplication, gene copies can evolve by conserving the same functions or undergoing mutations and becoming nonfunctional or assuming new functions, thus providing a putative new selective advantage in a new environment (Greub and Raoult, 2003; Walsh, 1995). Rickettsia prowazekii, the most 
reduced and degraded rickettsial genome that lacks the genes encoding the biosynthesis of purines and pyrimidines (Andersson et al., 1998), exhibits five copies of $t l c 1$ genes. These genes encode ADP/ATP translocases responsible of energy exploitation from host cells (Greub and Raoult, 2003; Renesto et al., 2005). Similar sequences were found in $R$. typhi, $R$. rickettsii and $R$. montanensis. Thus, the duplication of the tlc genes in Rickettsia is most likely explained by their important role in maintaining an efficient uptake and transport system of host cytoplasmic. ATP Four to 14 copies of spoT genes, involved in stringent response and the adaptation to intracellular environment, were also found in rickettsiae (Ogata et al., 2005a; Renesto et al., 2005; Rovery et al., 2005). The R. conorii genome has multiple copies of ampG agent encoding $\beta$-lactamase, which may explain the resistance of these bacteria to $\beta$ lactam antibiotics (Ogata, 2001). The T4SS, a multiple component, membrane-spanning transporter system containing eight distinct classes such as the MPF-T class (P-T4SSs), is largely found in many rickettsial genomes. Rickettsiae possess an incomplete P-T4SS system (related to systems of the IncP group conjugative plasmid) that is characterized by the lack of virB5 but the duplication of the virB4, virB6, virB8 and virB9 genes (Gillespie et al., 2016). The $R$. prowazekii genome has six Vir components (virB4, virB8-virB11, virD4), and the virB4 and virB9 were duplicated (Gillespie et al., 2009). Seventeen orthologous surface cell antigen-coding genes (sca) were identified in rickettsial genomes (Blanc, 2005). SCA proteins autotransporter proteins that were demonstrated to play roles in mammalian cell infection as well as infection of their arthropod host cells, notably by promoting actin-based motility (Sears et al., 2012). The $R$. bellii genome possesses a set of complete conjugation genes, and pilli like-filaments were observed on the bacterial surface (Ogata et al., 2006). Among 13 tested Rickettsia collection strains, 11 got positive conjugation gene detection. This suggests that the conjugation elements are widely present among Rickettsia spp (88), and that horizontal gene transfer (HGT) occured at a high rate (Weinert et al., 2009). Within amoebae, 
HGTs have given the Rickettsia ancestor the access to novel gene pools, with possibility to acquire foreign DNA from other intracellular bacteria, thus, in capability of adaptation environment (Ogata et al., 2006). In addition, a RAGE module, considered as a genetic exchange facilitator, was found in multiple copies in the genome from Rickettsia endosymbiont of Ixodes scapularis (REIS), the largest rickettsial genome described to date (Gillespie et al., 2014, 2012).

Finally, a large number of mobile genetic elements (MGEs) referred to as mobilome are found in rickettsiae despite their reduced genome size. This mobilome, mostly consisting of plasmids, may ensure DNA movement within and between genomes. To date, at least 20 known rickettsial plasmids have been described in 11 species despite their allopatric lifestyle (Diop et al., 2017). Recent phylogenomic analysis revealed that rickettsial plasmids are undergoing reductive evolutionary events similar to those affecting their co-residing chromosomes (El Karkouri et al., 2016). Rickettsial plasmids were thus shaped by a biphasic model of convergent evolution including a strong reductive evolution as well as an increased complexity via horizontal gene transfer and gene duplication and genesis (El Karkouri et al., 2016). The most reduced and virulent rickettsial genomes have probablely lost plasmid(s) during their evolution when compared to the related milder or non pathogenic species (Darby et al., 2007; El Karkouri et al., 2017; Ogata et al., 2005a).

\section{Conclusions and Perspectives}

Rickettsia species are strictly intracellular bacteria that are likely to have evolved from a presumably free-living ancestor and followed a transition to an obligate intracellular lifestyle. To adapt to such a bottleneck lifestyle associated with genetic drift, Rickettsia species have been shaped by distinct evolutionary processes resulting not only in differences in genome size, but also in genomic architecture. Generally, rickettsial genomes are small and contain a 
342 high ratio of non-coding DNA, which suggests that the reductive trend is their dominant mode

343 of evolution. Comparative sequence analysis has provided important clues on the mechanisms

344 driving the genome-reduction process of Rickettsia spp. This phenomenon is marked by a

345 selected loss of genes such as those associated with amino-acid, ATP, LPS and cell wall

346 component biosynthesis with a loss of regulatory genes and a high preservation of toxin-

347 associated proteins and toxin-antitoxin modules. Homologous intra-chromosomal

348 recombination, principal mechanism for genomic rearrangement structures seems play a role

349 in rapid gene loss. Consequently, rickettsiae have evolved under a distinct process including a

350 strong reductive evolution as well as a paradoxical expansion of genetic elements acquired by

351 horizontal gene transfer and gene duplication and genesis. Thus, during the course of

352 evolution, rickettsial genomes had a trend of gene loss rather than gene acquisition or

353 duplication, but these strong selective effects co-exist with functional duplications required

354 for survival. In order to understand the evolution of genome size and content, it is necessary

355 to understand the balance between proliferation and elimination of genetic material in these

356 intracellular bacteria. 
Andersson, J.O., Andersson, S.G., 1999. Genome degradation is an ongoing process in Rickettsia. Mol. Biol. Evol. 16, 1178-1191. https://doi.org/10.1093/oxfordjournals.molbev.a026208

Andersson, S.G.., Kurland, C.G., 1998. Reductive evolution of resident genomes. Trends Microbiol. 6, 263-268. https://doi.org/10.1016/S0966-842X(98)01312-2

Andersson, S.G., Stothard, D.R., Fuerst, P., Kurland, C.G., 1999. Molecular phylogeny and rearrangement of rRNA genes in Rickettsia species. Mol. Biol. Evol. 16, 987-995. https://doi.org/10.1093/oxfordjournals.molbev.a026188

Andersson, S.G., Zomorodipour, A., Andersson, J.O., Sicheritz-Pontén, T., Alsmark, U.C.M., Podowski, R.M., Näslund, A.K., Eriksson, A.-S., Winkler, H.H., Kurland, C.G., 1998. The genome sequence of Rickettsia prowazekii and the origin of mitochondria. Nature 396, 133-140.

Audia, J.P., Winkler, H.H., 2006. Study of the five Rickettsia prowazekii proteins annotated as ATP/ADP translocases (TIC): only TIc1 transports ATP/ADP, while Tlc4 and Tlc5 transport other ribonucleotides. J. Bacteriol. 188, 6261-6268. https://doi.org/10.1128/JB.00371-06

Baldridge, G.D., Burkhardt, N., Herron, M.J., Kurtti, T.J., Munderloh, U.G., 2005. Analysis of fluorescent protein expression in transformants of Rickettsia monacensis, an obligate intracellular tick symbiont. Appl. Environ. Microbiol. 71, 2095-2105. https://doi.org/10.1128/AEM.71.4.2095-2105.2005

Baldridge, G.D., Burkhardt, N.Y., Felsheim, R.F., Kurtti, T.J., Munderloh, U.G., 2007. Transposon insertion reveals pRM, a plasmid of Rickettsia monacensis. Appl. Environ. Microbiol. 73, 4984-4995. https://doi.org/10.1128/AEM.00988-07

Balraj, P., Karkouri, K.E., Vestris, G., Espinosa, L., Raoult, D., Renesto, P., 2008a. RickA Expression is not sufficient to promote actin-based motility of Rickettsia raoultii. PLoS ONE 3, e2582. https://doi.org/10.1371/journal.pone.0002582

Balraj, P., Nappez, C., Raoult, D., Renesto, P., 2008b. Western-blot detection of RickA within spotted fever group rickettsiae using a specific monoclonal antibody. FEMS Microbiol. Lett. 286, 257262. https://doi.org/10.1111/j.1574-6968.2008.01283.x

Balraj, P., Renesto, P., Raoult, D., 2009. Advances in Rickettsia pathogenicity. Ann. N. Y. Acad. Sci. 1166, 94-105. https://doi.org/10.1111/j.1749-6632.2009.04517.x

Bechah, Y., El Karkouri, K., Mediannikov, O., Leroy, Q., Pelletier, N., Robert, C., Medigue, C., Mege, J.L., Raoult, D., 2010. Genomic, proteomic, and transcriptomic analysis of virulent and avirulent Rickettsia prowazekii reveals its adaptive mutation capabilities. Genome Res. 20, 655-663. https://doi.org/10.1101/gr.103564.109

Blanc, G., 2005. Molecular evolution of Rickettsia surface antigens: evidence of positive selection. Mol. Biol. Evol. 22, 2073-2083. https://doi.org/10.1093/molbev/msi199

Blanc, G., Ogata, H., Robert, C., Audic, S., Claverie, J.-M., Raoult, D., 2007. Lateral gene transfer between obligate intracellular bacteria: evidence from the Rickettsia massiliae genome. Genome Res. 17, 1657-1664. https://doi.org/10.1101/gr.6742107

Blanc, G., Ogata, H., Robert, C., Audic, S., Suhre, K., Vestris, G., Claverie, J.-M., Raoult, D., 2007. Reductive genome evolution from the mother of Rickettsia. PLoS Genet 3, e14.

Brynnel, E.U., Kurland, C.G., Moran, N.A., Andersson, S.G., 1998. Evolutionary rates for tuf genes in endosymbionts of aphids. Mol. Biol. Evol. 15, 574-582.

Caturegli, P., Asanovich, K.M., Walls, J.J., Bakken, J.S., Madigan, J.E., Popov, V.L., Dumler, J.S., 2000. ankA: an Ehrlichia phagocytophila group gene encoding a cytoplasmic protein antigen with ankyrin repeats. Infect. Immun. 68, 5277-5283.

Cazalet, C., Rusniok, C., Brüggemann, H., Zidane, N., Magnier, A., Ma, L., Tichit, M., Jarraud, S., Bouchier, C., Vandenesch, F., Kunst, F., Etienne, J., Glaser, P., Buchrieser, C., 2004. Evidence in the Legionella pneumophila genome for exploitation of host cell functions and high genome plasticity. Nat. Genet. 36, 1165-1173. https://doi.org/10.1038/ng1447

Claverie, J.-M., Ogata, H., 2003. The insertion of palindromic repeats in the evolution of proteins. Trends Biochem. Sci. 28, 75-80. https://doi.org/10.1016/S0968-0004(02)00036-1 
Darby, A.C., Cho, N.-H., Fuxelius, H.-H., Westberg, J., Andersson, S.G.E., 2007. Intracellular pathogens go extreme: genome evolution in the Rickettsiales. Trends Genet. 23, 511-520. https://doi.org/10.1016/j.tig.2007.08.002

Diop, A., Raoult, D., Fournier, P.-E., 2017. Rickettsial genomics and the paradigm of genome reduction associated with increased virulence. Microbes Infect. https://doi.org/10.1016/j.micinf.2017.11.009

Dong, X., El Karkouri, K., Robert, C., Gavory, F., Raoult, D., Fournier, P.-E., 2012. Genomic comparison of Rickettsia helvetica and other Rickettsia species. J. Bacteriol. 194, 2751-2751. https://doi.org/10.1128/JB.00299-12

Dong, X., El Karkouri, K., Robert, C., Raoult, D., Fournier, P.-E., 2012. Genome Sequence of Rickettsia australis, the agent of queensland tick typhus. J. Bacteriol. 194, 5129. https://doi.org/10.1128/JB.01117-12

Dunning Hotopp, J.C., Lin, M., Madupu, R., Crabtree, J., Angiuoli, S.V., Eisen, J., Seshadri, R., Ren, Q., Wu, M., Utterback, T.R., Smith, S., Lewis, M., Khouri, H., Zhang, C., Niu, H., Lin, Q., Ohashi, N., Zhi, N., Nelson, W., Brinkac, L.M., Dodson, R.J., Rosovitz, M.J., Sundaram, J., Daugherty, S.C., Davidsen, T., Durkin, A.S., Gwinn, M., Haft, D.H., Selengut, J.D., Sullivan, S.A., Zafar, N., Zhou, L., Benahmed, F., Forberger, H., Halpin, R., Mulligan, S., Robinson, J., White, O., Rikihisa, Y., Tettelin, H., 2006. Comparative genomics of emerging human ehrlichiosis agents. PLoS Genet. 2, e21. https://doi.org/10.1371/journal.pgen.0020021

Eisen, J.A., Heidelberg, J.F., White, O., Salzberg, S.L., 2000. Evidence for symmetric chromosomal inversions around the replication origin in bacteria. Genome Biol. 1, research0011-1.

El Karkouri, K., Kowalczewska, M., Armstrong, N., Azza, S., Fournier, P.-E., Raoult, D., 2017. Multiomics analysis sheds light on the evolution and the intracellular lifestyle strategies of spotted fever group Rickettsia spp. Front. Microbiol. 8. https://doi.org/10.3389/fmicb.2017.01363

El Karkouri, K., Mediannikov, O., Robert, C., Raoult, D., Fournier, P.-E., 2016. Genome sequence of the tick-borne pathogen Rickettsia raoultii. Genome Announc. 4, e00157-16. https://doi.org/10.1128/genomeA.00157-16

Felsheim, R.F., Kurtti, T.J., Munderloh, U.G., 2009. Genome sequence of the endosymbiont Rickettsia peacockii and comparison with virulent Rickettsia rickettsii: identification of virulence factors. PLoS ONE 4, e8361. https://doi.org/10.1371/journal.pone.0008361

Fournier, P.-E., El Karkouri, K., Leroy, Q., Robert, C., Giumelli, B., Renesto, P., Socolovschi, C., Parola, P., Audic, S., Raoult, D., 2009. Analysis of the Rickettsia africae genome reveals that virulence acquisition in Rickettsia species may be explained by genome reduction. BMC Genomics 10, 166. https://doi.org/10.1186/1471-2164-10-166

Fraser, C.M., Gocayne, J.D., White, O., Adams, M.D., Clayton, R.A., Fleischmann, R.D., Bult, C.J., Kerlavage, A.R., Sutton, G., Kelley, J.M., Fritchman, R.D., Weidman, J.F., Small, K.V., Sandusky, M., Fuhrmann, J., Nguyen, D., Utterback, T.R., Saudek, D.M., Phillips, C.A., Merrick, J.M., Tomb, J.F., Dougherty, B.A., Bott, K.F., Hu, P.C., Lucier, T.S., Peterson, S.N., Smith, H.O., Hutchison, C.A., Venter, J.C., 1995. The minimal gene complement of Mycoplasma genitalium. Science 270, 397-403.

Fuxelius, H.-H., Darby, A., Min, C.-K., Cho, N.-H., Andersson, S.G.E., 2007. The genomic and metabolic diversity of Rickettsia. Res. Microbiol. 158, 745-753. https://doi.org/10.1016/j.resmic.2007.09.008

Georgiades, K., Raoult, D., 2011. Genomes of the most dangerous epidemic bacteria have a virulence repertoire characterized by fewer genes but more toxin-antitoxin modules. PLOS ONE 6, e17962. https://doi.org/10.1371/journal.pone.0017962

Gillespie, J.J., Ammerman, N.C., Dreher-Lesnick, S.M., Rahman, M.S., Worley, M.J., Setubal, J.C., Sobral, B.S., Azad, A.F., 2009. An Anomalous type IV secretion system in Rickettsia Is evolutionarily conserved. PLOS ONE 4, e4833. https://doi.org/10.1371/journal.pone.0004833

Gillespie, J.J., Beier, M.S., Rahman, M.S., Ammerman, N.C., Shallom, J.M., Purkayastha, A., Sobral, B.S., Azad, A.F., 2007. Plasmids and rickettsial evolution: insight from Rickettsia felis. PLoS ONE 2, e266. https://doi.org/10.1371/journal.pone.0000266 
Gillespie, J.J., Joardar, V., Williams, K.P., Driscoll, T., Hostetler, J.B., Nordberg, E., Shukla, M., Walenz, B., Hill, C.A., Nene, V.M., Azad, A.F., Sobral, B.W., Caler, E., 2012. A Rickettsia genome overrun by mobile genetic elements provides insight into the acquisition of genes characteristic of an obligate intracellular lifestyle. J. Bacteriol. 194, 376-394. https://doi.org/10.1128/JB.06244-11

Gillespie, J.J., Kaur, S.J., Rahman, M.S., Rennoll-Bankert, K., Sears, K.T., Beier-Sexton, M., Azad, A.F., 2014. Secretome of obligate intracellular Rickettsia. FEMS Microbiol. Rev. n/a-n/a. https://doi.org/10.1111/1574-6976.12084

Gillespie, J.J., Phan, I.Q.H., Driscoll, T.P., Guillotte, M.L., Lehman, S.S., Rennoll-Bankert, K.E., Subramanian, S., Beier-Sexton, M., Myler, P.J., Rahman, M.S., Azad, A.F., 2016. The Rickettsia type IV secretion system: unrealized complexity mired by gene family expansion. Pathog. Dis. 74, ftw058. https://doi.org/10.1093/femspd/ftw058

Gouin, E., Egile, C., Dehoux, P., Villiers, V., Adams, J., Gertler, F., Li, R., Cossart, P., 2004. The RickA protein of Rickettsia conorii activates the Arp2/3 complex. Nature 427, 457.

Gouin, E., Gantelet, H., Egile, C., Lasa, I., Ohayon, H., Villiers, V., Gounon, P., Sansonetti, P.J., Cossart, P., 1999. A comparative study of the actin-based motilities of the pathogenic bacteria Listeria monocytogenes, Shigella flexneri and Rickettsia conorii. J. Cell Sci. 112, 1697-1708.

Greub, G., Raoult, D., 2003. History of the ADP/ATP-translocase-encoding gene, a parasitism gene transferred from a Chlamydiales ancestor to plants 1 billion years ago. Appl. Environ. Microbiol. 69, 5530-5535. https://doi.org/10.1128/AEM.69.9.5530-5535.2003

Heinzen, R.A., Hayes, S.F., Peacock, M.G., Hackstadt, T., 1993. Directional actin polymerization associated with spotted fever group Rickettsia infection of vero cells. Infect. Immun. 61, 1926-1935.

Holste, D., Weiss, O., Grosse, I., Herzel, H., 2000. Are noncoding sequences of Rickettsia prowazekii remnants of "neutralized" genes? J. Mol. Evol. 51, 353-362. https://doi.org/10.1007/s002390010097

Hooper, S.D., 2003. On the nature of gene innovation: duplication patterns in microbial genomes. Mol. Biol. Evol. 20, 945-954. https://doi.org/10.1093/molbev/msg101

Hunter, D.J., Torkelson, J.L., Bodnar, J., Mortazavi, B., Laurent, T., Deason, J., Thephavongsa, K., Zhong, J., 2015. The Rickettsia endosymbiont of Ixodes pacificus contains all the genes of de novo folate biosynthesis. PloS One 10, e0144552.

Jeng, R.L., Goley, E.D., D'Alessio, J.A., Chaga, O.Y., Svitkina, T.M., Borisy, G.G., Heinzen, R.A., Welch, M.D., 2004. A Rickettsia WASP-like protein activates the Arp2/3 complex and mediates actinbased motility: Rickettsia RickA activates the Arp2/3 complex. Cell. Microbiol. 6, 761-769. https://doi.org/10.1111/j.1462-5822.2004.00402.x

Katoh, K., Standley, D.M., 2013. MAFFT multiple sequence alignment software Version 7: Improvements in performance and usability. Mol. Biol. Evol. 30, 772-780. https://doi.org/10.1093/molbev/mst010

Keeling, P.J., Charlebois, R.L., Ford Doolittle, W., 1994. Archaebacterial genomes: eubacterial form and eukaryotic content. Curr. Opin. Genet. Dev. 4, 816-822. https://doi.org/10.1016/0959437X(94)90065-5

Krawiec, S., Riley, M., 1990. Organization of the bacterial chromosome. Microbiol. Rev. 54, 502-539.

Lechner, M., Findeils s, S., Steiner, L., Marz, M., Stadler, P.F., Prohaska, S.J., 2011. Proteinortho: detection of (co-) orthologs in large-scale analysis. BMC Bioinformatics 12, 124.

McLeod, M.P., Qin, X., Karpathy, S.E., Gioia, J., Highlander, S.K., Fox, G.E., McNeill, T.Z., Jiang, H., Muzny, D., Jacob, L.S., Hawes, A.C., Sodergren, E., Gill, R., Hume, J., Morgan, M., Fan, G., Amin, A.G., Gibbs, R.A., Hong, C., Yu, X. -j., Walker, D.H., Weinstock, G.M., 2004. Complete Genome sequence of Rickettsia typhi and comparison with sequences of other rickettsiae. J. Bacteriol. 186, 5842-5855. https://doi.org/10.1128/JB.186.17.5842-5855.2004

Merhej, V., Georgiades, K., Raoult, D., 2013. Postgenomic analysis of bacterial pathogens repertoire reveals genome reduction rather than virulence factors. Brief. Funct. Genomics 12, 291-304. https://doi.org/10.1093/bfgp/elt015 
Merhej, V., Raoult, D., 2011. Rickettsial evolution in the light of comparative genomics. Biol. Rev. 86, 379-405. https://doi.org/10.1111/j.1469-185X.2010.00151.x

Merhej, V., Royer-Carenzi, M., Pontarotti, P., Raoult, D., 2009. Massive comparative genomic analysis reveals convergent evolution of specialized bacteria. Biol. Direct 4, 13. https://doi.org/10.1186/1745-6150-4-13

Moran, N.A., 2002. Microbial minimalism: genome reduction in bacterial pathogens. Cell $108,583-$ 586.

Munson, M.A., Baumann, L., Baumann, P., 1993. Buchnera aphidicola (a prokaryotic endosymbiont of aphids) contains a putative $16 \mathrm{~S}$ rRNA operon unlinked to the $23 \mathrm{~S}$ rRNA-encoding gene: sequence determination, and promoter and terminator analysis. Gene 137, 171-178. https://doi.org/10.1016/0378-1119(93)90003-L

Murray, G.G.R., Weinert, L.A., Rhule, E.L., Welch, J.J., 2016. The phylogeny of Rickettsia using different evolutionary signatures: how tree-like is bacterial evolution? Syst. Biol. 65, 265279. https://doi.org/10.1093/sysbio/syv084

Ogata, H., 2005. Rickettsia felis, from culture to genome sequencing. Ann. N. Y. Acad. Sci. 1063, 2634. https://doi.org/10.1196/annals.1355.004

Ogata, H., 2001. Mechanisms of evolution in Rickettsia conorii and R. prowazekii. Science 293, 20932098. https://doi.org/10.1126/science.1061471

Ogata, H., Audic, S., Abergel, C., Fournier, P.-E., Claverie, J.-M., 2002. Protein coding palindromes are a unique but recurrent feature in Rickettsia. Genome Res. 12, 808-816.

Ogata, H., Audic, S., Barbe, V., Artiguenave, F., Fournier, P.-E., Raoult, D., M Claverie, J., 2000. Selfish DNA in protein-coding genes of Rickettsia.

Ogata, H., La Scola, B., Audic, S., Renesto, P., Blanc, G., Robert, C., Fournier, P.-E., Claverie, J.-M., Raoult, D., 2006. Genome sequence of Rickettsia bellii illuminates the role of amoebae in gene exchanges between intracellular pathogens. PLoS Genet. 2, e76. https://doi.org/10.1371/journal.pgen.0020076

Ogata, H., Renesto, P., Audic, S., Robert, C., Blanc, G., Fournier, P.-E., Parinello, H., Claverie, J.-M., Raoult, D., 2005a. The genome sequence of Rickettsia felis identifies the first putative conjugative plasmid in an obligate intracellular parasite. PLoS Biol. 3, e248. https://doi.org/10.1371/journal.pbio.0030248

Ogata, H., Suhre, K., Claverie, J.-M., 2005b. Discovery of protein-coding palindromic repeats in Wolbachia. Trends Microbiol. 13, 253-5. https://doi.org/10.1016/j.tim.2005.03.013

Papenfort, K., Vanderpool, C.K., 2015. Target activation by regulatory RNAs in bacteria. FEMS Microbiol. Rev. 39, 362-378. https://doi.org/10.1093/femsre/fuv016

Parola, P., Paddock, C.D., Socolovschi, C., Labruna, M.B., Mediannikov, O., Kernif, T., Abdad, M.Y., Stenos, J., Bitam, I., Fournier, P.-E., Raoult, D., 2013. Update on tick-borne rickettsioses around the World: a geographic approach. Clin. Microbiol. Rev. 26, 657-702. https://doi.org/10.1128/CMR.00032-13

Raoult, D., Roux, V., 1997. Rickettsioses as paradigms of new or emerging infectious diseases. Clin. Microbiol. Rev. 10, 694-719.

Reed, S.C.O., Lamason, R.L., Risca, V.I., Abernathy, E., Welch, M.D., 2014. Rickettsia actin-based motility occurs in distinct phases mediated by different actin nucleators. Curr. Biol. 24, 98103. https://doi.org/10.1016/j.cub.2013.11.025

Renesto, P., Ogata, H., Audic, S., Claverie, J.-M., Raoult, D., 2005. Some lessons from Rickettsia genomics. FEMS Microbiol. Rev. 29, 99-117. https://doi.org/10.1016/j.femsre.2004.09.002

Riegler, M., Iturbe-Ormaetxe, I., Woolfit, M., Miller, W.J., O'Neill, S.L., 2012. Tandem repeat markers as novel diagnostic tools for high resolution fingerprinting of Wolbachia. BMC Microbiol. 12, S12.

Rogozin, I.B., Makarova, K.S., Natale, D.A., Spiridonov, A.N., Tatusov, R.L., Wolf, Y.I., Yin, J., Koonin, E.V., 2002. Congruent evolution of different classes of non-coding DNA in prokaryotic genomes. Nucleic Acids Res. 30, 4264-4271. 
Rovery, C., Renesto, P., Crapoulet, N., Matsumoto, K., Parola, P., Ogata, H., Raoult, D., 2005. Transcriptional response of Rickettsia conorii exposed to temperature variation and stress starvation. Res. Microbiol. 156, 211-218. https://doi.org/10.1016/j.resmic.2004.09.002

Sahni, S.K., Narra, H.P., Sahni, A., Walker, D.H., 2013. Recent molecular insights into rickettsial pathogenesis and immunity. Future Microbiol. 8, 1265-1288. https://doi.org/10.2217/fmb.13.102

Sakharkar, K.R., 2004. Genome reduction in prokaryotic obligatory intracellular parasites of humans: a comparative analysis. Int. J. Syst. Evol. Microbiol. 54, 1937-1941. https://doi.org/10.1099/ijs.0.63090-0

Schroeder, C.L.C., Narra, H.P., Rojas, M., Sahni, A., Patel, J., Khanipov, K., Wood, T.G., Fofanov, Y., Sahni, S.K., 2015. Bacterial small RNAs in the genus Rickettsia. BMC Genomics 16. https://doi.org/10.1186/s12864-015-2293-7

Sears, K.T., Ceraul, S.M., Gillespie, J.J., Allen, E.D., Popov, V.L., Ammerman, N.C., Rahman, M.S., Azad, A.F., 2012. Surface proteome analysis and characterization of surface cell antigen (Sca) or autotransporter family of Rickettsia typhi. PLoS Pathog. 8, e1002856. https://doi.org/10.1371/journal.ppat.1002856

Seemann, T., 2014. Prokka: rapid prokaryotic genome annotation. Bioinformatics 30, 2068-2069. https://doi.org/10.1093/bioinformatics/btu153

Seshadri, R., Paulsen, I.T., Eisen, J.A., Read, T.D., Nelson, K.E., Nelson, W.C., Ward, N.L., Tettelin, H., Davidsen, T.M., Beanan, M.J., others, 2003. Complete genome sequence of the Q-fever pathogen Coxiella burnetii. Proc. Natl. Acad. Sci. 100, 5455-5460.

Sicheritz-Pontén, T., Andersson, S.G., 1997. GRS: a graphic tool for genome retrieval and segment analysis. Microb. Comp. Genomics 2, 123-139.

Shpynov, S.N., Fournier, P.E., Pozdnichenko, N.N., Gumenuk, A.S., Skiba, A.A., 2018. New approaches in the systematics of rickettsiae. New Microbes New Infect. 23, 93-102. https://doi.org10.1016/j.nmni.2018.02.012.

Stothard, D.R., Clark, J.B., Fuerst, P.A., 1994. Ancestral divergence of Rickettsia bellii from the spotted fever and typhus groups of Rickettsia and antiquity of the genus Rickettsia. Int. J. Syst. Evol. Microbiol. 44, 798-804.

Syvänen, A.-C., Amiri, H., Jamal, A., Andersson, S.G., Kurland, C.G., 1996. A chimeric disposition of the elongation factor genes in Rickettsia prowazekii. J. Bacteriol. 178, 6192-6199.

Tamura, K., Stecher, G., Peterson, D., Filipski, A., Kumar, S., 2013. MEGA6: Molecular evolutionary genetics analysis version 6.0. Mol. Biol. Evol. 30, 2725-2729. https://doi.org/10.1093/molbev/mst197

Walker, D.H., 2005. Progress in rickettsial genome analysis from pioneering of Rickettsia prowazekii to the recent Rickettsia typhi. Ann. N. Y. Acad. Sci. 1063, 13-25. https://doi.org/10.1196/annals.1355.003

Walsh, J.B., 1995. How often do duplicated genes evolve new functions? Genetics 139, 421-428.

Weinert, L.A., Welch, J.J., Jiggins, F.M., 2009. Conjugation genes are common throughout the genus Rickettsia and are transmitted horizontally. Proc. R. Soc. B Biol. Sci. 276, 3619-3627. https://doi.org/10.1098/rspb.2009.0875

Weinert, L.A., Werren, J.H., Aebi, A., Stone, G.N., Jiggins, F.M., 2009. Evolution and diversity of Rickettsia bacteria. BMC Biol. 7, 6. https://doi.org/10.1186/1741-7007-7-6

Wixon, J., 2001. Featured organism: reductive evolution in bacteria: Buchnera sp., Rickettsia prowazekii and Mycobacterium leprae. Comp. Funct. Genomics 2, 44-48.

Wolf, Y.I., Koonin, E.V., 2013. Genome reduction as the dominant mode of evolution: prospects \& overviews. BioEssays 35, 829-837. https://doi.org/10.1002/bies.201300037

Woodard, A., Wood, D.O., 2011. Analysis of convergent gene transcripts in the obligate intracellular bacterium Rickettsia prowazekii. PLoS ONE 6, e16537. https://doi.org/10.1371/journal.pone.0016537

Wu, M., Sun, L.V., Vamathevan, J., Riegler, M., Deboy, R., Brownlie, J.C., McGraw, E.A., Martin, W., Esser, C., Ahmadinejad, N., Wiegand, C., Madupu, R., Beanan, M.J., Brinkac, L.M., Daugherty, 
S.C., Durkin, A.S., Kolonay, J.F., Nelson, W.C., Mohamoud, Y., Lee, P., Berry, K., Young, M.B., Utterback, T., Weidman, J., Nierman, W.C., Paulsen, I.T., Nelson, K.E., Tettelin, H., O'Neill, S.L., Eisen, J.A., 2004. Phylogenomics of the reproductive parasite Wolbachia pipientis wMel: A streamlined genome overrun by mobile genetic elements. PLoS Biol. 2, e69. https://doi.org/10.1371/journal.pbio.0020069

Xin, D., El Karkouri, K., Robert, C., Raoult, D., Fournier, P.-E., 2012. Genomic comparison of Rickettsia honei strain RBT and other Rickettsia Species. J. Bacteriol. 194, 4145. https://doi.org/10.1128/JB.00802-12 
623 Figure 1: Phylogenomic tree of 29 Rickettsia species based on whole-genome sequence

624

625

626

627

628

629

630

631

632

633

634

635

636

637

638

639

640

641

642

643

644 analysis using the Maximum Likehood method within the FastTree software. Genomes were aligned using Mugsy software. Values at the nodes are percentages. Numbers at the nodes represent the percentages of bootstrap values obtained by repeating the analysis 1000 times to generate a majority consensus tree. Only values greater than $90 \%$ were reported. $\mathrm{AG}=$ Ancestral group TG $=$ Typhus group TRG $=$ Transitional group $; \mathrm{SFG}=$ Spotted fever group .

Figure 2: Phylogenomic tree based on 591 core proteins and pathogenic and genomic features, of Rickettsia species exhibiting various degrees of pathogenesis. For each genome (downloaded from GenBank), gene prediction was obtained using the Prokka software (Seemann, 2014). The core genome was identified using the ProteinOrtho software (Lechner et al., 2011). Then, the amino acid sequences of 591 proteins (Supplementary Table) conserved in all studied genomes were concatenated for each species and multiple alignment was performed using the Mafft software (Katoh and Standley, 2013, p. 2). Gapped positions were removed. The phylogenetic inferences were obtained using the Maximum Likelihood method and the MEGA software version 6 (Tamura et al., 2013). Branching support was evaluated using the bootstrap method with 1000 replications. Bootstrap values greater than $90 \%$ are shown at the nodes. Properties of each species were extracted from the following references (Andersson et al., 1998; G. Blanc et al., 2007; Guillaume Blanc et al., 2007b; El Karkouri et al., 2017, 2016; Fournier et al., 2009; McLeod et al., 2004; Ogata, 2001b; Ogata et al., 2006, 2005a). NA = data not available; RPEs = Rickettsia palindromic elements. 


\begin{tabular}{|c|c|c|}
\hline Clusters ID & Protein function & Genes \\
\hline cRIG00001 & rnpA Ribonuclease P & rnpA \\
\hline cRIG00002 & rplT $50 \mathrm{~S}$ ribosomal protein $\mathrm{L}$ & $\mathrm{rpl}$ \\
\hline cRIG00003 & nrdG Organic radical activating enzymes & nrd \\
\hline cRIG00004 & rplY 50S ribosomal protein $\mathrm{L}$ & $\mathrm{rpl}$ \\
\hline cRIG00005 & ychF GTP-binding protein $\mathrm{YchF}$ & $y \operatorname{chF}$ \\
\hline cRIG00006 & murE UDP-N-acetylmuramoylalanyl-D-glutamate-- , 2,6-diaminopimelate ligase & mur \\
\hline cRIG00007 & $\begin{array}{l}\text { murF UDP-N-acetylmuramoylalanyl-D-glutamyl- , 2,6-diaminopimelate--D-alanyl-D-alanyl } \\
\text { ligase }\end{array}$ & mur \\
\hline cRIG00008 & mraY1 Phospho-N-acetylmuramoyl-pentapeptide- transferase & $\operatorname{mraY}$ \\
\hline cRIG00009 & recG ATP-dependent DNA helicase RecG & rec \\
\hline cRIG00010 & traX F pilin acetylation protein TraX & $\operatorname{tra}$ \\
\hline cRIG00011 & mviN Integral membrane protein MviN & mviN \\
\hline cRIG00012 & ppa Inorganic pyrophosphatase & ppa \\
\hline cRIG00013 & ccmE Cytochrome c-type biogenesis protein ccmE & $\mathrm{ccm}$ \\
\hline cRIG00014 & Sco2 protein precursor & Sco2 \\
\hline cRIG00015 & secD Protein-export membrane protein secD & $\mathrm{sec}$ \\
\hline cRIG00016 & yajC Preprotein translocase YajC subunit & yajC \\
\hline cRIG00017 & gyrB DNA gyrase subunit B & gyr \\
\hline cRIG00018 & murA UDP-N-acetylglucosamine -1-carboxyvinyltransferase & mur \\
\hline cRIG00019 & surA Parvulin-like peptidyl-prolyl isomerase & surA \\
\hline cRIG00020 & secA Preprotein translocase secA subunit & $\mathrm{sec}$ \\
\hline cRIG00021 & Unknown & - \\
\hline cRIG00022 & uvrC Excinuclease $\mathrm{ABC}$ subunit $\mathrm{C}$ & uvr \\
\hline cRIG00023 & cmk Cytidylate kinase & cmk \\
\hline cRIG00024 & rpsA $30 \mathrm{~S}$ ribosomal protein $\mathrm{S} 1$ & $\mathrm{rps}$ \\
\hline cRIG00025 & clpP ATP-dependent Clp protease proteolytic subunit & $\mathrm{clp}$ \\
\hline cRIG00026 & glmU UDP-N-acetylglucosamine pyrophosphorylase & $\operatorname{glm} U$ \\
\hline cRIG00027 & Unknown & - \\
\hline cRIG00028 & Unknown & - \\
\hline cRIG00029 & lpdA2 Dihydrolipoamide dehydrogenase & $\operatorname{lpdA}$ \\
\hline cRIG00030 & 5 -Formyltetrahydrofolate cyclo-ligase & - \\
\hline cRIG00031 & rnd Ribonuclease D & rnd \\
\hline cRIG00032 & gcvT Glycine cleavage T-protein & gcvT \\
\hline cRIG00033 & putP Na+/proline symporter and signal transduction histidine kinase & putP \\
\hline cRIG00034 & hemC Porphobilinogen deaminase & hemC \\
\hline cRIG00035 & trpS Tryptophanyl-tRNA synthetase & $\operatorname{trpS}$ \\
\hline cRIG00036 & plsC 1-acyl-sn-glycerol--phosphate acyltransferase & plsC \\
\hline cRIG00037 & ampG1 AmpG & ampG \\
\hline cRIG00038 & tlc3 ATP/ADP translocase & tlc \\
\hline cRIG00039 & Unknown & - \\
\hline cRIG00040 & ispB Octaprenyl-diphosphate synthase & isp \\
\hline cRIG00041 & potE Putrescine-ornithine antiporter & potE \\
\hline cRIG00042 & iscA2 Iron-sulfur cluster assembly accessory protein & isc \\
\hline
\end{tabular}




\begin{tabular}{|c|c|c|}
\hline cRIG00043 & iscU FeS cluster assembly scaffold IscU & isc \\
\hline cRIG00044 & iscS Cysteine desulfurase IscS & isc \\
\hline cRIG00045 & sp11 NifS-like protein & spl1 \\
\hline cRIG00046 & Unknown & - \\
\hline cRIG00047 & ntrY Nitrogen regulation protein NtrY & ntr \\
\hline cRIG00048 & rpsU 30S ribosomal protein $\mathrm{S}$ & rps \\
\hline cRIG00049 & Unknown & - \\
\hline cRIG00050 & ileS Isoleucyl-tRNA synthetase & ileS \\
\hline cRIG00051 & accC Acetyl-CoA carboxylase, biotin carboxylase & accC \\
\hline cRIG00052 & pccB Propionyl-CoA carboxylase beta chain precursor & pccB \\
\hline cRIG00053 & aas2 -acylglycerophosphoethanolamine acyltransferase & aas \\
\hline cRIG00054 & znuB Zinc/manganese $\mathrm{ABC}$ transporter permease protein & znuB \\
\hline cRIG00055 & ubiG Ubiquinone biosynthesis O-methyltransferase & ubi \\
\hline cRIG00056 & gltX Glutamyl-tRNA synthetase & gltX \\
\hline cRIG00057 & groEL 60 kD chaperonin & groEL \\
\hline cRIG00058 & groES $10 \mathrm{kD}$ chaperonin & groES \\
\hline cRIG00059 & rph Ribonuclease PH & $\mathrm{rph}$ \\
\hline cRIG00060 & grpE GrpE protein & grpE \\
\hline cRIG00061 & perM Permease PerM-like protein & perM \\
\hline cRIG00062 & DnaA-like protein & DnaA \\
\hline cRIG00063 & rplQ 50S ribosomal protein L17 & $\mathrm{rpl}$ \\
\hline cRIG00064 & rpoA DNA-directed RNA polymerase alpha chain & rpo \\
\hline cRIG00065 & rpsK $30 \mathrm{~S}$ ribosomal protein $\mathrm{S} 11$ & rps \\
\hline cRIG00066 & rpsM 30S ribosomal protein S13 & rps \\
\hline cRIG00067 & adk Adenylate kinase & adk \\
\hline cRIG00068 & secY Preprotein translocase secY subunit & $\sec$ \\
\hline cRIG00069 & rplO 50S ribosomal protein $\mathrm{L} 15$ & $\mathrm{rpl}$ \\
\hline cRIG00070 & rpmD 50S ribosomal protein $\mathrm{L} 30$ & rpm \\
\hline cRIG00071 & rpsE 30S ribosomal protein S5 & rps \\
\hline cRIG00072 & rplR 50S riboSomal protein L18 & $\mathrm{rpl}$ \\
\hline cRIG00073 & rplF 50S riboSomal protein L6 & $\mathrm{rpl}$ \\
\hline cRIG00074 & rp30SH 30S riboSomal protein S8 & rps \\
\hline cRIG00075 & rp30SN 30S riboSomal protein S14 & rps \\
\hline cRIG00076 & rplE 50S riboSomal protein L5 & $\mathrm{rpl}$ \\
\hline cRIG00077 & rplX 50S riboSomal protein L24 & $\mathrm{rpl}$ \\
\hline cRIG00078 & rplN 50S ribosomal protein L14 & $\mathrm{rpl}$ \\
\hline cRIG00079 & rpsQ 30S ribosomal protein S17 & rps \\
\hline cRIG00080 & rpmC 50S ribosomal protein L29 & $\mathrm{rpm}$ \\
\hline cRIG00081 & rplP 50S ribosomal protein L16 & $\mathrm{rpl}$ \\
\hline cRIG00082 & rpsC $30 \mathrm{~S}$ ribosomal protein $\mathrm{S} 3$ & rps \\
\hline cRIG00083 & rplV 50S ribosomal protein L22 & $\mathrm{rpl}$ \\
\hline cRIG00084 & rpsS 30S ribosomal protein S19 & rps \\
\hline cRIG00085 & rplB 50S ribosomal protein L2 & $\mathrm{rpl}$ \\
\hline cRIG00086 & rplW 50S ribosomal protein L23 & $\mathrm{rpl}$ \\
\hline cRIG00087 & rplD 50S ribosomal protein L4 & $\mathrm{rpl}$ \\
\hline cRIG00088 & rpsJ 30S ribosomal protein $\mathrm{S} 10$ & rps \\
\hline cRIG00089 & tuf Elongation factor EF-Tu & tuf \\
\hline cRIG00090 & fumC Fumarate hydratase & fumC \\
\hline
\end{tabular}




\begin{tabular}{|c|c|c|}
\hline cRIG00091 & ftsZ Cell division protein ftsZ & fts \\
\hline cRIG00092 & NifU-like protein & NifU \\
\hline cRIG00093 & ampG2 AmpG & ampG \\
\hline cRIG00094 & rhlE ATP-dependent RNA helicase RhlE & rhlE \\
\hline cRIG00095 & cspA Cold shock-like protein & $\operatorname{csp} A$ \\
\hline cRIG00096 & ksgA Dimethyladenosine transferase & $\mathrm{ksgA}$ \\
\hline cRIG00097 & Unknown & - \\
\hline cRIG00098 & ostA Organic solvent tolerance protein-like protein & ostA \\
\hline cRIG00099 & xseA Exodeoxyribonuclease VII, large subunit & xse \\
\hline cRIG00100 & xth2 Exodeoxyribonuclease III & xth \\
\hline cRIG00101 & GTP-binding protein & - \\
\hline cRIG00102 & ubiB 2-polyprenylphenol -hydroxylase & ubi \\
\hline cRIG00103 & ubiE Ubiquinone/menaquinone biosynthesis methlytransferase UbiE & ubi \\
\hline cRIG00104 & Unknown & - \\
\hline cRIG00105 & tatD Putative deoxyribonuclease TatD & tat \\
\hline cRIG00106 & metG Methionyl-tRNA synthetase & metG \\
\hline cRIG00107 & tmk1Thymidylate kinase & tmk1 \\
\hline cRIG00108 & proP4 Proline/betaine transporter & proP4 \\
\hline cRIG00109 & ubiA 4-hydroxybenzoate octaprenyltransferase & ubi \\
\hline cRIG00110 & valS Valyl-tRNA synthetase & vals \\
\hline cRIG00111 & $\mathrm{RmuC}$ family protein & \\
\hline cRIG00112 & exsB Trans-regulatory protein ExsB & $\begin{array}{c}\text { RmuC } \\
\text { exsB }\end{array}$ \\
\hline cRIG00113 & msbA2 Multidrug resistance protein & $\mathrm{msbA}$ \\
\hline cRIG00114 & Unknown & - \\
\hline cRIG00115 & bcr2 MFS-type bicyclomycin resistance protein & bcr2 \\
\hline cRIG00116 & Lipoprotein releasing system, transmembrane protein, LolC/E family protein & Lol \\
\hline cRIG00117 & lolD Lipoprotein releasing system ATP-binding protein LolD & Lol \\
\hline cRIG00118 & Unknown & - \\
\hline cRIG00119 & Hemolysin-like protein & - \\
\hline cRIG00120 & ccmF Cytochrome c-type biogenesis protein ccmF & $\mathrm{ccm}$ \\
\hline cRIG00121 & ompB, sca5 Outer membrane protein rOmpB & sca \\
\hline cRIG00122 & Beta-glucosidase & - \\
\hline cRIG00123 & lpxK Tetraacyldisaccharide 4'-kinase & lpx \\
\hline cRIG00124 & ligA DNA ligase, NAD-dependent & $\operatorname{lig}$ \\
\hline cRIG00125 & tgt Queuine tRNA-ribosyltransferase & $\operatorname{tgt}$ \\
\hline cRIG00126 & $\mathrm{ABC}$ transporter substrate binding protein & - \\
\hline cRIG00127 & NADHubiquinone oxidoreductase $17,2 \mathrm{kD}$ subunit & - \\
\hline cRIG00128 & rnhA Ribonuclease $\mathrm{H}$ & $\mathrm{rnh}$ \\
\hline cRIG00129 & Unknown & - \\
\hline cRIG00130 & Unknown & - \\
\hline cRIG00131 & coaE Dephospho-CoA kinase & $\mathrm{coaE}$ \\
\hline cRIG00132 & dnaQ DNA polymerase III epsilon chain & dna \\
\hline cRIG00133 & surf1 Surfeit locus protein & surf1 \\
\hline cRIG00134 & ATP-dependent helicase & - \\
\hline cRIG00135 & fabD Malonyl CoA-acyl carrier protein transacylase & fab \\
\hline cRIG00136 & tlc5 ATP/ADP translocase & tlc \\
\hline cRIG00137 & tlyC Hemolysin C & tlyC \\
\hline
\end{tabular}




\begin{tabular}{|c|c|c|}
\hline cRIG00138 & Putative metal-dependent hydrolase & - \\
\hline cRIG00139 & lipA Lipoic acid synthetase & lip \\
\hline cRIG00140 & glyA Glycine/serine hydroxymethyltransferase & gly \\
\hline cRIG00141 & Serine esterase & - \\
\hline cRIG00142 & nth Endonuclease III & nth \\
\hline cRIG00143 & Putative methyltransferase & - \\
\hline cRIG00144 & ABC-type transport systems periplasmic component & - \\
\hline cRIG00145 & tatA Twin-arginine translocation protein TatA & tat \\
\hline cRIG00146 & pgpA Phosphatidylglycerophosphatase A & pgpA \\
\hline cRIG00147 & rplU 50S ribosomal protein $\mathrm{L} 21$ & $\mathrm{rpl}$ \\
\hline cRIG00148 & rpmA 50S ribosomal protein L27 & rpm \\
\hline cRIG00149 & Unknown & - \\
\hline cRIG00150 & proP5 Proline/betaine transporter & proP \\
\hline cRIG00151 & NAD-specific glutamate dehydrogenase & NAD \\
\hline cRIG00152 & trmE tRNA modification GTPase TrmE & trm \\
\hline cRIG00153 & recA $\operatorname{Rec} A$ & rec \\
\hline cRIG00154 & fabG 3-oxoacyl reductase & fab \\
\hline cRIG00155 & acpP Acyl carrier protein & acpP \\
\hline cRIG00156 & fabF 3-oxoacyl- & fab \\
\hline cRIG00157 & mreC Rod shape-determining protein $\mathrm{MreC}$ & mre \\
\hline cRIG00158 & mreB Rod shape-determining protein MreB & mre \\
\hline cRIG00159 & Putative permeases & - \\
\hline cRIG00160 & pal Peptidoglycan-associated lipoprotein precursor & pal \\
\hline cRIG00161 & rpmF $50 \mathrm{~S}$ ribosomal protein $\mathrm{L} 32$ & $\mathrm{rpm}$ \\
\hline cRIG00162 & smpA tmRNA-binding protein & smpA \\
\hline cRIG00163 & ftsY Signal recognition particle-docking protein FtsY & $\mathrm{fts}$ \\
\hline cRIG00164 & polA DNA polymerase I & pol \\
\hline cRIG00165 & dnaE DNA polymerase III alpha chain & dna \\
\hline cRIG00166 & udg UDP-glucose 6-dehydrogenase & udg \\
\hline cRIG00167 & Unknown & - \\
\hline cRIG00168 & ampG3 AmpG & ampG \\
\hline cRIG00169 & tatC Sec-independent protein translocase protein TatC & tat \\
\hline cRIG00170 & serS Seryl-tRNA synthetase & $\operatorname{ser} S$ \\
\hline cRIG00171 & virB4-2 VirB4 & virB \\
\hline cRIG00172 & Unknown & - \\
\hline cRIG00173 & terC Tellurium resistance protein TerC & terC \\
\hline cRIG00174 & nuoL1 NADH dehydrogenase I chain L & nuo \\
\hline cRIG00175 & nuoM NADH dehydrogenase I chain M & nuo \\
\hline cRIG00176 & ccmA Heme exporter protein A & $\mathrm{ccm}$ \\
\hline cRIG00177 & nuoI NADH dehydrogenase I chain I & nuo \\
\hline cRIG00178 & nuoH NADH dehydrogenase I chain $\mathrm{H}$ & nuo \\
\hline cRIG00179 & nuoG NADH dehydrogenase I chain G & nuo \\
\hline cRIG00180 & atpG ATP synthase gamma chain & atp \\
\hline cRIG00181 & atpA ATP synthase alpha chain & atp \\
\hline cRIG00182 & atpH ATP synthase delta chain & atp \\
\hline cRIG00183 & lpdA1 Dihydrolipoamide dehydrogenase & lpdA \\
\hline cRIG00184 & Unknown & - \\
\hline cRIG00185 & kefB Glutathione-regulated potassium-efflux system protein KefB & kefB \\
\hline
\end{tabular}




\begin{tabular}{|c|c|c|}
\hline cRIG00186 & Iojap-related protein & lojap \\
\hline cRIG00187 & bolA2 BolA-like protein & bolA \\
\hline cRIG00188 & infA Translation initiation factor IF- & $\inf$ \\
\hline cRIG00189 & maf Nucleotide-binding protein implicated in inhibition of septum formation & maf \\
\hline cRIG00190 & dksA DnaK suppressor-like protein & dksA \\
\hline cRIG00191 & xerC Tyrosine recombinase XerC & xerC \\
\hline cRIG00192 & hypothetical protein & - \\
\hline cRIG00193 & ftsK Cell division protein FtsK & fts \\
\hline cRIG00194 & mraY2 Undecaprenyl-phosphate alpha-N-acetylglucosaminyltransferase & $\operatorname{mraY}$ \\
\hline cRIG00195 & Unknown & - \\
\hline cRIG00196 & Unknown & - \\
\hline cRIG00197 & Putative outer surface protein & sca \\
\hline cRIG00198 & fdxA Ferredoxin & fdxA \\
\hline cRIG00199 & ccmC Heme exporter protein $\mathrm{C}$ & $\mathrm{ccm}$ \\
\hline cRIG00200 & Cation diffusion facilitator family transporter & - \\
\hline cRIG00201 & omp $17 \mathrm{kD}$ surface antigen precursor & Sca \\
\hline cRIG00202 & znuC Zinc ABC transporter ATP-binding protein & znu \\
\hline cRIG00203 & uvrA Excinuclease $\mathrm{ABC}$ subunit $\mathrm{A}$ & uvr \\
\hline cRIG00204 & ssb Single-stranded DNA-binding protein & ssb \\
\hline cRIG00205 & DAP dipeptidyl aminopeptidase/acylaminoacyl-peptidase-like protein & dap \\
\hline cRIG00206 & htpG Heat shock protein htpG & htpG \\
\hline cRIG00207 & hemA 5-aminolevulinic acid synthase & hemA \\
\hline cRIG00208 & tig Trigger factor & tig \\
\hline cRIG00209 & obg GTP-binding protein & obg \\
\hline cRIG00210 & gltA Citrate synthase I & gltA \\
\hline cRIG00211 & Uracil-DNA glycosylase, family & Uracil \\
\hline cRIG00212 & Ribosomal large subunit pseudouridine synthases RluD subfamily protein & RluD \\
\hline cRIG00213 & hemK Methylase of polypeptide chain release factors & hemK \\
\hline cRIG00214 & Sua5/YciO/YrdC/YwlC family putative translation factor protein & Sua5 \\
\hline cRIG00215 & glyS Glycyl-tRNA synthetase beta chain & gly \\
\hline cRIG00216 & glyQ Glycyl-tRNA synthetase alpha chain & gly \\
\hline cRIG00217 & Unknown & - \\
\hline cRIG00218 & Unknown & - \\
\hline cRIG00219 & dnaG DNA primase & dna \\
\hline cRIG00220 & sec59 Dolichol kinase & $\mathrm{sec}$ \\
\hline cRIG00221 & greA Transcription elongation factor GreA & greA \\
\hline cRIG00222 & pntA2 NAD $(\mathrm{P})$ transhydrogenase subunit alpha & pnt \\
\hline cRIG00223 & pntA1 NAD $(\mathrm{P})$ transhydrogenase subunit alpha & pnt \\
\hline cRIG00224 & lolA Outer membrane lipoprotein-sorting protein LolA & Lol \\
\hline cRIG00225 & Unknown & - \\
\hline cRIG00226 & Putative aspartyl protease & - \\
\hline cRIG00227 & glnQ Glutamine $\mathrm{ABC}$ transporter ATP-binding protein & $\operatorname{gln}$ \\
\hline cRIG00228 & phnP Metal-dependent hydrolases of the beta-lactamase superfamily I & phnP \\
\hline cRIG00229 & Unknown & - \\
\hline cRIG00230 & Putative permeases & - \\
\hline cRIG00231 & holC DNA polymerase III chi subunit HolC & holC \\
\hline cRIG00232 & dapE Succinyl-diaminopimelate desuccinylase & dap \\
\hline cRIG00233 & Unknown & - \\
\hline
\end{tabular}




\begin{tabular}{|c|c|c|}
\hline cRIG00234 & lipB Putative lipoate-protein ligase B & lip \\
\hline cRIG00235 & $\mathrm{rpsP} 30 \mathrm{~S}$ ribosomal protein $\mathrm{S} 16$ & $\mathrm{rps}$ \\
\hline cRIG00236 & mutL DNA mismatch repair protein MutL & mut \\
\hline cRIG00237 & proP7 Proline/betaine transporter & proP \\
\hline cRIG00238 & hemF Coproporphyrinogen III oxidase precursor & hemF \\
\hline cRIG00239 & Putative membrane protein & - \\
\hline cRIG00240 & hemH Putative ferrochelatase & hemH \\
\hline cRIG00241 & hemE Uroporphyrinogen decarboxylase & hemE \\
\hline cRIG00242 & Unknown & - \\
\hline cRIG00243 & trxA Thioredoxin & $\operatorname{trx}$ \\
\hline cRIG00244 & rfbE O-antigen export system ATP-binding protein RfbE & $\mathrm{rfb}$ \\
\hline cRIG00245 & rfbA O-antigen export system permease protein $\mathrm{RfbA}$ & $\mathrm{rfb}$ \\
\hline cRIG00246 & gltD NADPH-dependent glutamate synthase beta chain and related oxidoreductases & gltA \\
\hline cRIG00247 & lpxA Acyl- & $\operatorname{lpx}$ \\
\hline cRIG00248 & fabZ (3R)-hydroxymyristoyl- & fab \\
\hline cRIG00249 & lpxD UDP-3-O- & lpx \\
\hline cRIG00250 & Putative P-loop hydrolase & - \\
\hline cRIG00251 & znuA Zinc/manganese $\mathrm{ABC}$ transporter substrate binding protein & znu \\
\hline cRIG00252 & pcnB Poly(A) polymerase & $\mathrm{pcnB}$ \\
\hline cRIG00253 & atpF ATP synthase B chain & atp \\
\hline cRIG00254 & atpX ATP synthase B chain & atp \\
\hline cRIG00255 & atpE ATP synthase $\mathrm{C}$ chain & atp \\
\hline cRIG00256 & atpB ATP synthase A chain & atp \\
\hline cRIG00257 & Unknown & - \\
\hline cRIG00258 & dsbG Protein-disulfide isomerase & $\mathrm{dsbG}$ \\
\hline cRIG00259 & Transcriptional regulator & - \\
\hline cRIG00260 & Unknown & - \\
\hline cRIG00261 & cvpA Putative colicin V production membrane protein & $\operatorname{cvpA}$ \\
\hline cRIG00262 & clpB ClpB & clp \\
\hline cRIG00263 & hypothetical protein & - \\
\hline cRIG00264 & rpsF 30S ribosomal protein S6 & rps \\
\hline cRIG00265 & rpsR30 S ribosomal protein S18 & rps \\
\hline cRIG00266 & rplI 50S ribosomal protein L9 & $\mathrm{rpl}$ \\
\hline cRIG00267 & tilS, mesJ tRNA(Ile)-lysidine synthetase & tils \\
\hline cRIG00268 & ftsH ATP-dependent metalloprotease FtsH & fts \\
\hline cRIG00269 & sdhB Succinate dehydrogenase iron-sulfur protein & sdh \\
\hline cRIG00270 & Unknown & - \\
\hline cRIG00271 & lgt Prolipoprotein diacylglyceryl transferase & lgt \\
\hline cRIG00272 & Putative membrane protein & - \\
\hline cRIG00273 & yidC Preprotein translocase subunit YidC & yidC \\
\hline cRIG00274 & pgsA CDP-diacylglycerol--glycerol-3-phosphate 3-phosphatidyltransferase & pgsA \\
\hline cRIG00275 & Unknown & - \\
\hline cRIG00276 & tlc1 ATP/ADP translocase & tlc \\
\hline cRIG00277 & uhpC Sugar phosphate permease & uhpC \\
\hline cRIG00278 & ndk Nucleoside diphosphate kinase & ndk \\
\hline cRIG00279 & gidA Glucose-inhibited division protein A & gid \\
\hline cRIG00280 & soj ATPase involved in chromosome partitioning & soj \\
\hline cRIG00281 & ParB-like partition proteins & ParB \\
\hline
\end{tabular}




\begin{tabular}{|c|c|c|}
\hline cRIG00282 & abcTABC transporter ATP-binding protein & $\mathrm{abcT}$ \\
\hline cRIG00283 & Unknown & - \\
\hline cRIG00284 & kdsA -deoxy--phosphooctulonate synthase & $\mathrm{kds}$ \\
\hline cRIG00285 & iscAIron-sulfur cluster assembly accessory protein & isc \\
\hline cRIG00286 & dgt Deoxyguanosinetriphosphate triphosphohydrolase & dgt \\
\hline cRIG00287 & $\operatorname{argS~Arginyl-tRNA~synthetase~}$ & $\arg$ \\
\hline cRIG00288 & Unknown & - \\
\hline cRIG00289 & parC Topoisomerase IV subunit A & Par \\
\hline cRIG00290 & Unknown & - \\
\hline cRIG00291 & dcd Deoxycytidine triphosphate deaminase & ded \\
\hline cRIG00292 & secB Protein-export protein secB & $\mathrm{sec}$ \\
\hline cRIG00293 & czcR Transcriptional activator protein CzcR & czcR \\
\hline cRIG00294 & GTP cyclohydrolase I & GTP \\
\hline cRIG00295 & Unknown & - \\
\hline cRIG00296 & pntB NAD(p) transhydrogenase subunit beta & pnt \\
\hline cRIG00297 & ompW OmpW family outer-membrane protein & Sca \\
\hline cRIG00298 & sam S-adenosylmethionine transporter & sam \\
\hline cRIG00299 & proP1 Proline/betaine transporter & proP \\
\hline cRIG00300 & cysS Cysteinyl-tRNA synthetase & cysS \\
\hline cRIG00301 & rpsB 30S ribosomal protein $\mathrm{S} 2$ & rps \\
\hline cRIG00302 & tsf Elongation factor EF-Ts & tsf \\
\hline cRIG00303 & kdtA 3-deoxy-D-manno-octulosonic-acid transferase & kdtA \\
\hline cRIG00304 & Unknown & - \\
\hline cRIG00305 & aatA Aspartate aminotransferase A & aatA \\
\hline cRIG00306 & Unknown & - \\
\hline cRIG00307 & vacJ VacJ lipoprotein precursor & vacJ \\
\hline cRIG00308 & ABC-type transporter related to toluene tolerance & - \\
\hline cRIG00309 & alr Alanine racemase & alr \\
\hline cRIG00310 & $\mathrm{ABC}$ transporter permease protein & - \\
\hline cRIG00311 & mkl Ribonucleotide ABC transporter ATP-binding protein & $\mathrm{mkl}$ \\
\hline cRIG00312 & rpmB 50S ribosomal protein $\mathrm{L} 28$ & $\mathrm{rpm}$ \\
\hline cRIG00313 & rpmE 50S ribosomal protein $\mathrm{L} 31$ & $\mathrm{rpm}$ \\
\hline cRIG00314 & Hypothetical GTP-binding protein & - \\
\hline cRIG00315 & virB3 VirB3 & virB \\
\hline cRIG00316 & virB4-1 VirB4 & virB \\
\hline cRIG00317 & virB6-3 VirB6 & virB \\
\hline cRIG00318 & virB6-4 VirB6 & virB \\
\hline cRIG00319 & virB6-5 VirB6 & virB \\
\hline cRIG00320 & trmD tRNA (guanine-n1)-methyltransferase & trm \\
\hline cRIG00321 & rplS 50S ribosomal protein L19 & $\mathrm{rpl}$ \\
\hline cRIG00322 & Unknown & - \\
\hline cRIG00323 & secF Protein-export membrane protein secF & $\sec$ \\
\hline cRIG00324 & nuoF NADH dehydrogenase I chain F & nuo \\
\hline cRIG00325 & lepB Signal peptidase I & lepB \\
\hline cRIG00326 & era GTP-binding protein Era & era \\
\hline cRIG00327 & ruvC Crossover junction endodeoxyribonuclease RuvC & ruvC \\
\hline cRIG00328 & Putative nucleoside-diphosphate-sugar epimerase & - \\
\hline cRIG00329 & mrp Mrp & $\operatorname{mrp}$ \\
\hline
\end{tabular}




\begin{tabular}{|c|c|c|}
\hline cRIG00330 & hflK Protease activity modulator HflK & hfl \\
\hline cRIG00331 & hflC2 Membrane protease subunit, stomatin/prohibitin-like protein & $\mathrm{hfl}$ \\
\hline cRIG00332 & htrA Periplasmic serine protease & htrA \\
\hline cRIG00333 & Putative sulfurtransferase & - \\
\hline cRIG00334 & sdhC Succinate dehydrogenase cytochrome b-556 subunit & sdh \\
\hline cRIG00335 & yqiY Amino acid $\mathrm{ABC}$ transporter permease protein & yqi \\
\hline cRIG00336 & rpsL 30S ribosomal protein S12 & rps \\
\hline cRIG00337 & rpsG 30S ribosomal protein $\mathrm{S} 7$ & rps \\
\hline cRIG00338 & fusA Elongation factor EF-G & fusA \\
\hline cRIG00339 & nusG Transcription antitermination protein NusG & nus \\
\hline cRIG00340 & rplK 50S ribosomal protein L11 & $\mathrm{rpl}$ \\
\hline cRIG00341 & rplA 50S ribosomal protein L1 & $\mathrm{rpl}$ \\
\hline cRIG00342 & rplJ 50S ribosomal protein L10 & $\mathrm{rpl}$ \\
\hline cRIG00343 & rplL 50S ribosomal protein L7/L12 & $\mathrm{rpl}$ \\
\hline cRIG00344 & rpoB DNA-directed RNA polymerase beta chain & rpo \\
\hline cRIG00345 & rpoC DNA-directed RNA polymerase beta prime chain & rpo \\
\hline cRIG00346 & pepA Aminopeptidase A & pepA \\
\hline cRIG00347 & Chromosome partitioning protein-like protein & - \\
\hline cRIG00348 & aspS Aspartyl-tRNA synthetase & aspS \\
\hline cRIG00349 & Integral membrane protein, interacts with FtsH & fts \\
\hline cRIG00350 & yqiX Amino acid $\mathrm{ABC}$ transporter substrate binding protein & yqi \\
\hline cRIG00351 & gatA Glutamyl-tRNA(Gln) amidotransferase subunit $\mathrm{A}$ & gat \\
\hline cRIG00352 & gatC Glutamyl-tRNA(Gln) amidotransferase subunit $\mathrm{C}$ & gat \\
\hline cRIG00353 & rrf Ribosome recycling factor & $\operatorname{rrf}$ \\
\hline cRIG00354 & pyrH Uridylate kinase & pyr \\
\hline cRIG00355 & mnhE Multisubunit $\mathrm{Na}+\mathrm{H}+$ antiporter, MnhE subunit & $\mathrm{mnh}$ \\
\hline cRIG00356 & emrB MFS-type multidrug resistance protein B (SPLIT GENE) & emrB \\
\hline cRIG00357 & omp1 Outer membrane protein omp & Sca \\
\hline cRIG00358 & Putative membrane-associated zinc metalloprotease & - \\
\hline cRIG00359 & nusB $N$ utilization substance protein $B$ & nus \\
\hline cRIG00360 & rrmJ Ribosomal RNA large subunit methyltransferase J & $\mathrm{rrmJ}$ \\
\hline cRIG00361 & Oligoketide cyclase/lipid transport protein & - \\
\hline cRIG00362 & Unknown & - \\
\hline cRIG00363 & hupA DNA-binding protein HU & hupA \\
\hline cRIG00364 & holB DNA polymerase III delta subunit & hol \\
\hline cRIG00365 & ffh Signal recognition particle protein & ffh \\
\hline cRIG00366 & gltP Na+/H+-dicarboxylate symporters & glt \\
\hline cRIG00367 & Putative 6-pyruvoyl tetrahydropterin synthase & - \\
\hline cRIG00368 & sucB Dihydrolipoamide acetyltransferase component & suc \\
\hline cRIG00369 & sucA2 -oxoglutarate dehydrogenase Ecomponent & suc \\
\hline cRIG00370 & recN DNA repair protein $\mathrm{RecN}$ & rec \\
\hline cRIG00371 & comL DNA uptake lipoprotein & comL \\
\hline cRIG00372 & dnaJ DnaJ & dna \\
\hline cRIG00373 & dnaK DnaK & dna \\
\hline cRIG00374 & Heat shock protease & hsl \\
\hline cRIG00375 & Unknown & - \\
\hline cRIG00376 & holA DNA polymerase III, delta subunit & hol \\
\hline cRIG00377 & coq7 Ubiquinone biosynthesis protein coq & $\operatorname{coq} 7$ \\
\hline
\end{tabular}




\begin{tabular}{|c|c|c|}
\hline cRIG00378 & coxC Cytochrome c oxidase subunit III & $\operatorname{cox}$ \\
\hline cRIG00379 & virB2 VirB2-like protein & $\operatorname{virB}$ \\
\hline cRIG00380 & RafdapD 2,3,4,5-tetrahydropyridine-2-carboxylate $\mathrm{N}$-succinyltransferase & dap \\
\hline cRIG00381 & uspA Universal stress protein UspA and related nucleotide-binding proteins & uspA \\
\hline cRIG00382 & imp TRAP-type uncharacterized transport system, periplasmic component & imp \\
\hline cRIG00383 & Unknown & - \\
\hline cRIG00384 & hscA Heat shock protein hscA & hsc \\
\hline cRIG00385 & rnhB Ribonuclease HII & $\mathrm{rnh}$ \\
\hline cRIG00386 & uvrB Excinuclease $\mathrm{ABC}$ subunit $\mathrm{B}$ & uvr \\
\hline cRIG00387 & grxC1 Glutaredoxin, GrxC family & $\operatorname{grxC} 1$ \\
\hline cRIG00388 & atm1 Multidrug resistance protein Atm & atm1 \\
\hline cRIG00389 & gyrA DNA gyrase subunit A & gyr \\
\hline cRIG00390 & def1 Polypeptide deformylase & def \\
\hline cRIG00391 & fmt Methionyl-tRNA formyltransferase & fmt \\
\hline cRIG00392 & abcT3 Multidrug resistance ABC transporter ATP-binding protein & abcT \\
\hline cRIG00393 & Unknown & - \\
\hline cRIG00394 & cydA Cytochrome d ubiquinol oxidase subunit I & cyd \\
\hline cRIG00395 & thrS Threonyl-tRNA synthetase & thrS \\
\hline cRIG00396 & Unknown & - \\
\hline cRIG00397 & tolC Type I secretion outer membrane protein TolC & tol \\
\hline cRIG00398 & Unknown & - \\
\hline cRIG00399 & Ankyrin repeat & - \\
\hline cRIG00400 & parE DNA topoisomerase IV, B subunit & Par \\
\hline cRIG00401 & ctp Carboxyl-terminal protease & ctp \\
\hline cRIG00402 & barA Histidine kinase sensor protein & barA \\
\hline cRIG00403 & Unknown & - \\
\hline cRIG00404 & Unknown & - \\
\hline cRIG00405 & WD40-like repeat & - \\
\hline cRIG00406 & rplM 50S ribosomal protein L13 & $\mathrm{rpl}$ \\
\hline cRIG00407 & rpsI 30S ribosomal protein $\mathrm{S} 9$ & rps \\
\hline cRIG00408 & nudH (Di)nucleoside polyphosphate hydrolase & nudH \\
\hline cRIG00409 & Regulatory components of sensory transduction system & - \\
\hline cRIG00410 & efp Translation elongation factor EF-P & efp \\
\hline cRIG00411 & suhB Extragenic suppressor protein suhB & suhB \\
\hline cRIG00412 & Unknown & - \\
\hline cRIG00413 & psd Phosphatidylserine decarboxylase & psd \\
\hline cRIG00414 & pssA CDP-diacylglycerol--serine O-phosphatidyltransferase & pssA \\
\hline cRIG00415 & hypothetical protein & - \\
\hline cRIG00416 & Unknown & - \\
\hline cRIG00417 & bolA1 BolA-like protein & bolA \\
\hline cRIG00418 & EAL domain containing protein & EAL \\
\hline cRIG00419 & murC UDP-N-acetylmuramate--alanine ligase & mur \\
\hline cRIG00420 & murB UDP-N-acetylenolpyruvoylglucosamine reductase & mur \\
\hline cRIG00421 & ddlB D-alanine--D-alanine ligase & ddlB \\
\hline cRIG00422 & Membrane protein implicated in regulation of membrane protease activity & - \\
\hline cRIG00423 & cycM Cytochrome c & сусM \\
\hline cRIG00424 & lpxC UDP-3-O- & $\operatorname{lpx}$ \\
\hline cRIG00425 & xth1 Exodeoxyribonuclease III & xth \\
\hline
\end{tabular}




\begin{tabular}{|c|c|c|}
\hline cRIG00426 & pdhA Pyruvate dehydrogenase e1 component, alpha subunit precursor & $\mathrm{pdh}$ \\
\hline cRIG00427 & pdhB Pyruvate dehydrogenase E1 component, beta subunit precursor & pdh \\
\hline cRIG00428 & typA GTP-binding protein TypA & typ \\
\hline cRIG00429 & hlpA Outer membrane protein & hlp \\
\hline cRIG00430 & icd Isocitrate dehydrogenase, NADP-dependent & icd \\
\hline cRIG00431 & Monovalent cation/proton antiporter, MnhG/PhaG subunit & MnhG \\
\hline cRIG00432 & mnhB Multisubunit Na+/H+ antiporter, MnhB subunit & $\mathrm{mnh}$ \\
\hline cRIG00433 & ccmB Heme exporter protein B & $\mathrm{ccm}$ \\
\hline cRIG00434 & Unknown & - \\
\hline cRIG00435 & petA Ubiquinol-cytochrome c reductase, iron-sulfur subunit & pet \\
\hline cRIG00436 & petB Cytochrome b & pet \\
\hline cRIG00437 & fbcH Cytochrome $\mathrm{c} 1$, heme protein precursor & $\mathrm{fbcH}$ \\
\hline cRIG00438 & nuoL2 NADH dehydrogenase I chain L & nuo \\
\hline cRIG00439 & nuoN2 NADHubiquinone oxidoreductase subunit (chain N) & nuo \\
\hline cRIG00440 & mnhC Multisubunit $\mathrm{Na}+\mathrm{H}+$ antiporter, MnhC subunit & $\mathrm{mnh}$ \\
\hline cRIG00441 & virB8-1VirB8 & virB \\
\hline cRIG00442 & virB8-2 VirB8 & $\operatorname{virB}$ \\
\hline cRIG00443 & virB9-2 VirB9 & $\operatorname{virB}$ \\
\hline cRIG00444 & virD4 VirD4 & $\operatorname{virD}$ \\
\hline cRIG00445 & gppA Guanosine pentaphosphate phosphohydrolase & gppA \\
\hline cRIG00446 & Unknown & - \\
\hline cRIG00447 & Unknown & - \\
\hline cRIG00448 & cysQ 3'(2'),'5-bisphosphate nucleotidase & cysQ \\
\hline cRIG00449 & mutS DNA mismatch repair protein MutS & mut \\
\hline cRIG00450 & lacA Ribose-5-phosphate isomerase & lacA \\
\hline cRIG00451 & nlpD1 Membrane-bound metallopeptidase & nlpD \\
\hline cRIG00452 & thyX Thymidylate synthase, flavin-dependent & thy \\
\hline cRIG00453 & tolB TolB protein precursor & tol \\
\hline cRIG00454 & hypothetical protein & - \\
\hline cRIG00455 & cox11, ctaG Cytochrome c oxidase assembly protein cox 11 & $\operatorname{cox}$ \\
\hline cRIG00456 & trmU tRNA (5-methylaminomethyl-2-thiouridylate)-methyltransferase & $\operatorname{trm}$ \\
\hline cRIG00457 & atrC1 Cationic amino acid transporter-1 & $\operatorname{atrC}$ \\
\hline cRIG00458 & hisS Histidyl-tRNA synthetase & his $S$ \\
\hline cRIG00459 & tolQ TolQ & tol \\
\hline cRIG00460 & tolR TolR & tol \\
\hline cRIG00461 & Periplasmic protein TonB, links inner and outer membranes & Ton \\
\hline cRIG00462 & proP10 Proline/betaine transporter & proP \\
\hline cRIG00463 & HlyD family secretion protein & HlyD \\
\hline cRIG00464 & aprD Alkaline protease secretion ATP-binding protein AprD & aprD \\
\hline cRIG00465 & asd Aspartate-semialdehyde dehydrogenase & asd \\
\hline cRIG00466 & Unknown & - \\
\hline cRIG00467 & hslV Heat shock protein HslV & hsl \\
\hline cRIG00468 & hslU Heat shock protein HslVU, ATPase subunit HslU & hsl \\
\hline cRIG00469 & lpxB Lipid-A-disaccharide synthase & $\operatorname{lpx}$ \\
\hline cRIG00470 & Aminodeoxychorismate lyase & - \\
\hline cRIG00471 & cyaY CyaY & cyaY \\
\hline cRIG00472 & gltX1 Glutamyl-tRNA synthetase & glt \\
\hline cRIG00473 & topA DNA topoisomerase I & top \\
\hline
\end{tabular}




\begin{tabular}{|c|c|c|}
\hline cRIG00474 & tdpX1 Thioredoxin peroxidase & $\operatorname{tdpX}$ \\
\hline cRIG00475 & hflC1 Membrane protease subunit, stomatin/prohibitin-like protein & hfl \\
\hline cRIG00476 & Putative membrane-associated metal-dependent hydrolase & - \\
\hline cRIG00477 & Efflux transporter, RND family, MFP subunit & rnd \\
\hline cRIG00478 & Putative hydrolase/acyltransferase & - \\
\hline cRIG00479 & Glycosyltransferase & - \\
\hline cRIG00480 & rpsD 30S ribosomal protein $\mathrm{S} 4$ & rps \\
\hline cRIG00481 & cyoB, ctaB Protoheme IX farnesyltransferase & суоB \\
\hline cRIG00482 & rimM 16S rRNA processing protein RimM & rimM \\
\hline cRIG00483 & Unknown & - \\
\hline cRIG00484 & xseB Exodeoxyribonuclease VII small subunit & xse \\
\hline cRIG00485 & mpg DNA-3-methyladenine glycosidase & mpg \\
\hline cRIG00486 & nuoE NADH dehydrogenase I chain E & nuo \\
\hline cRIG00487 & nuoD NADH dehydrogenase I chain D & nuo \\
\hline cRIG00488 & nuoC NADH dehydrogenase I chain C & nuo \\
\hline cRIG00489 & nuoA NADH dehydrogenase I chain A & nuo \\
\hline cRIG00490 & cutE Apolipoprotein N-acyltransferase & cut \\
\hline cRIG00491 & lysS Lysyl-tRNA synthetase & lys \\
\hline cRIG00492 & Putative permease & - \\
\hline cRIG00493 & tme Malate oxidoreductase & tme \\
\hline cRIG00494 & proP3 Proline/betaine transporter & proP \\
\hline cRIG00495 & mdh Malate dehydrogenase & mdh \\
\hline cRIG00496 & tlc2 ATP/ADP translocase & tlc \\
\hline cRIG00497 & pyrG CTP synthase & pyr \\
\hline cRIG00498 & kdsB 3-deoxy-manno-octulosonate cytidylyltransferase & $\mathrm{kds}$ \\
\hline cRIG00499 & folE GTP cyclohydrolase I & fol \\
\hline cRIG00500 & proS Prolyl-tRNA synthetase & pros \\
\hline cRIG00501 & ruvB Holliday junction DNA helicase RuvB & ruv \\
\hline cRIG00502 & msbA1 Multidrug resistance protein & $\mathrm{msbA}$ \\
\hline cRIG00503 & dacF Penicillin-binding protein dacF precursor & dac \\
\hline cRIG00504 & rlpA Rare lipoprotein A precursor & rlpA \\
\hline cRIG00505 & osmY Putative periplasmic or secreted lipoprotein & osmY \\
\hline cRIG00506 & ispZ Intracellular septation protein A & isp \\
\hline cRIG00507 & FTR1 family protein & FTR1 \\
\hline cRIG00508 & Unknown & - \\
\hline cRIG00509 & Unknown & - \\
\hline cRIG00510 & Protocatechuate-3,4-dioxygenase, beta subunit & - \\
\hline cRIG00511 & tlpA Thioldisulfide interchange protein tlpA & $\operatorname{tlpA}$ \\
\hline cRIG00512 & sppA1 Signal peptide peptidase SppA, 36K type & sppA \\
\hline cRIG00513 & dut Deoxyuridine 5'-triphosphate nucleotidohydrolase & dut \\
\hline cRIG00514 & mltESoluble lytic murein transglycosylase precursor & mltE \\
\hline cRIG00515 & mccF Microcin C7 self-immunity protein & $\mathrm{mccF}$ \\
\hline cRIG00516 & RecB family exonuclease & rec \\
\hline cRIG00517 & Putative glutamine amidotransferase & - \\
\hline cRIG00518 & coxA Cytochrome $\mathrm{c}$ oxidase polypeptide I & $\operatorname{cox}$ \\
\hline cRIG00519 & coxB Cytochrome c oxidase polypeptide II & $\operatorname{cox}$ \\
\hline cRIG00520 & nlpD2 Membrane-bound metallopeptidase & nlpD \\
\hline cRIG00521 & ftsW Cell division protein $\mathrm{ftsW}$ & $\mathrm{fts}$ \\
\hline
\end{tabular}




\begin{tabular}{|c|c|c|}
\hline cRIG00522 & $\begin{array}{l}\text { murG UDP-N-acetylglucosamine--N-acetylmuramyl- (pentapeptide) pyrophosphoryl- } \\
\text { undecaprenol N-acetylglucosamine transferase }\end{array}$ & mur \\
\hline cRIG00523 & Unknown & - \\
\hline cRIG00524 & Glycosyltransferase & - \\
\hline cRIG00525 & dapF Diaminopimelate epimerase & dap \\
\hline cRIG00526 & MiaB-like tRNA modifying enzyme & Mia \\
\hline cRIG00527 & pheS Phenylalanyl-tRNA synthetase alpha chain & phe \\
\hline cRIG00528 & pheT Phenylalanyl-tRNA synthetase beta chain & phe \\
\hline cRIG00529 & dnaN DNA polymerase III beta chain & dna \\
\hline cRIG00530 & Unknown & - \\
\hline cRIG00531 & rbfA Ribosome-binding factor A & $\mathrm{rbf}$ \\
\hline cRIG00532 & Putative membrane protein & - \\
\hline cRIG00533 & RDD family protein & rdd \\
\hline cRIG00534 & recR Recombination protein RecR & rec \\
\hline cRIG00535 & ppnK Putative inorganic polyphosphate/ATP-NAD kinase & ppnk \\
\hline cRIG00536 & Putative hydrolase of the metallo-beta-lactamase superfamily & - \\
\hline cRIG00537 & gpsA Glycerol--phosphate dehydrogenase & pgsA \\
\hline cRIG00538 & Putative permease & - \\
\hline cRIG00539 & Putative hydrolase/acyltransferase & - \\
\hline cRIG00540 & trxB1 Thioredoxin reductase & $\operatorname{trx}$ \\
\hline cRIG00541 & lgtD Glycosyl transferase & $\operatorname{lgt}$ \\
\hline cRIG00542 & uvrD DNA helicase II & uvr \\
\hline cRIG00543 & Unknown & - \\
\hline cRIG00544 & tdcB Threonine dehydratase & $\operatorname{tdcB}$ \\
\hline cRIG00545 & lon1 ATP-dependent protease La & lon1 \\
\hline cRIG00546 & yhbH Putative sigma(54) modulation protein & yhbH \\
\hline cRIG00547 & folD Methylenetetrahydrofolate dehydrogenase & fol \\
\hline cRIG00548 & $\operatorname{trxB} 2$ Thioredoxin reductase & $\operatorname{trx}$ \\
\hline cRIG00549 & nrdA Ribonucleoside-diphosphate reductase alpha chain & nrd \\
\hline cRIG00550 & nrdB Ribonucleoside-diphosphate reductase beta chain & nrd \\
\hline cRIG00551 & Unknown & - \\
\hline cRIG00552 & kpsF KpsF & $\mathrm{kpsF}$ \\
\hline cRIG00553 & pnp Polyribonucleotide nucleotidyltransferase & pnp \\
\hline cRIG00554 & rpsO 30S ribosomal protein $\mathrm{S} 15$ & rps \\
\hline cRIG00555 & truB tRNA pseudouridine synthase B & $\operatorname{truB}$ \\
\hline cRIG00556 & tlc4 ATP/ADP translocase & tlc \\
\hline cRIG00557 & sca Cell surface antigen Sca & Sca \\
\hline cRIG00558 & glnA Glutamine synthetase & $\operatorname{gln}$ \\
\hline cRIG00559 & Unknown & - \\
\hline cRIG00560 & ppdK Pyruvate,phosphate dikinase precursor & ppdk \\
\hline cRIG00561 & Glutathione S-transferase & - \\
\hline cRIG00562 & folC Folylpolyglutamate synthase & fol \\
\hline cRIG00563 & sodB Superoxide dismutase & sodB \\
\hline cRIG00564 & rssA Putative esterase of the alpha/beta hydrolase superfamily protein & rssA \\
\hline cRIG00565 & birA Biotin-(acetyl-CoA carboxylase) ligase & birA \\
\hline cRIG00566 & rho Transcription termination factor & rho \\
\hline cRIG00567 & mraZ MraZ protein & mra \\
\hline cRIG00568 & mraW S-adenosyl-methyltransferase MraW & mra \\
\hline
\end{tabular}




\begin{tabular}{lcc}
\hline cRIG00569 & ftsL Cell division protein FtsL & fts \\
cRIG00570 & pbpA2 Penicillin-binding protein & pbpA \\
cRIG00571 & pbpA1 Penicillin-binding protein & pbpA \\
cRIG00572 & Unknown & - \\
cRIG00573 & Unknown & - \\
cRIG00574 & ntrX Nitrogen assimilation regulatory protein NtrX & ntr \\
cRIG00575 & ubiH 2-polyprenyl-6-methoxyphenol 4-hydroxylase & ubi \\
cRIG00576 & nusA & ntilization substance protein A, transcription termination factor NusA \\
cRIG00577 & infB Translation initiation factor IF-2 & inf \\
cRIG00578 & Putative glycoprotein endopeptidase & - \\
cRIG00579 & Unknown & - \\
cRIG00580 & N6-adenine-specific methylase & N6 \\
cRIG00581 & dnaB Replicative DNA helicase & rluA \\
cRIG00582 & rluA2 Ribosomal large subunit pseudouridine synthase & dna \\
cRIG00583 & priA Primosomal protein N' & ubi \\
cRIG00584 & ubiX 3-octaprenyl-4-hydroxybenzoate carboxy-lyase & priA \\
cRIG00585 & Unknown & hemB \\
cRIG00586 & hemB Delta-aminolevulinic acid dehydratase & - \\
cRIG00587 & nuoN1 NADHubiquinone oxidoreductase subunit (chain N) \\
cRIG00588 & proP6 Proline/betaine transporter & nuo \\
cRIG00589 & dus Putative dihydrouridine synthase Dus & proP \\
cRIG00590 & phbC Poly-beta-hydroxybutyrate polymerase & dus \\
cRIG00591 & Unknown & phb \\
\hline
\end{tabular}




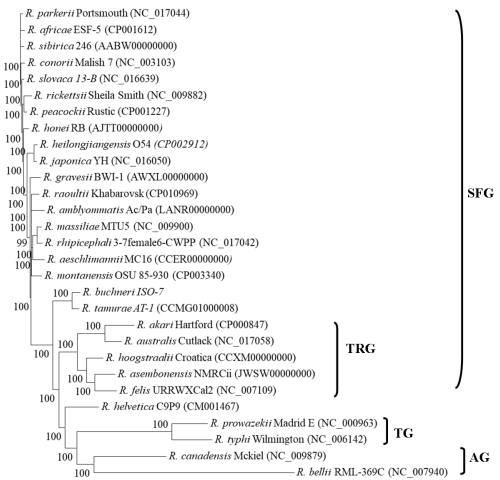




\begin{tabular}{|c|c|c|c|c|c|c|}
\hline Virulence & Size (bp) & $\begin{array}{c}\text { protein- } \\
\text { coding genes }\end{array}$ & $\begin{array}{l}\% \text { coding } \\
\text { s sequences }\end{array}$ & RNAs & pseudogenes & RPES \\
\hline Milder & $\begin{array}{c}1,278,540 \\
\text { pRaf:12,377 }\end{array}$ & 1112 & 78.26 & 39 & 246 & 460 \\
\hline Virulent & $1,250,021$ & 1083 & 77.76 & 36 & 200 & $\mathbf{N A}$ \\
\hline Virulent & $1,268,755$ & 1374 & 81.5 & 39 & 252 & 559 \\
\hline Virulent & $1,257,710$ & 1345 & 78.5 & 36 & 233 & NA \\
\hline Mild & $\begin{array}{c}1,360,898 \\
\text { pRma: } 15,286\end{array}$ & 968 & 69 & 39 & 286 & 562 \\
\hline Mild & $\begin{array}{c}1,344,605 \\
\text { pRra1:20,840 }\end{array}$ & 1180 & 71.2 & 39 & 339 & NA \\
\hline Mild & $\begin{array}{c}\text { pRra2:83,219 } \\
\text { pRra3:34,583 } \\
1,485,148 \\
\text { pRF:6,282 } \\
\text { pRF5:39,268 }\end{array}$ & 1444 & 83.8 & 39 & 130 & 726 \\
\hline $\begin{array}{l}\text { Highly } \\
\text { virulent }\end{array}$ & $1,111,523$ & 834 & 76.2 & 39 & 181 & 120 \\
\hline Virulent & $1,111,496$ & 838 & 76.3 & 39 & 185 & 121 \\
\hline Unknown & $1,522,076$ & 1429 & 82.5 & 40 & 100 & 526 \\
\hline
\end{tabular}

